SCiEnTia Marina 71(3)

September 2007, 605-622, Barcelona (Spain)

ISSN: 0214-8358

\title{
Relationships between deep-water polychaete fauna and environmental factors in the southeastern Gulf of California, Mexico
}

\author{
NURIA MÉNDEZ \\ Unidad Académica Mazatlán. Instituto de Ciencias del Mar y Limnología, Universidad Nacional Autónoma de México. \\ P.O. Box 811, Mazatlán 82000, Sinaloa, México.E-mail: nuri@ola.icmyl.unam.mx
}

\begin{abstract}
SUMMARY: Polychaetes inhabiting deep-sea soft bottoms in the southeastern Gulf of California were collected during four oceanographic cruises in 2000 and 2001 at a depth range of $732-2110 \mathrm{~m}$. A total of 84 taxa, representing 32 families, were collected. The relationships between density, species number and diversity and depth, dissolved oxygen, temperature, granulometry and organic matter content in sediments were analysed. Cluster analyses and MDS plots based on density allowed faunal assemblages to be established for each environmental variable: 700-1100 m, 1100-1600 m, 1900-2100 m and 2100$2200 \mathrm{~m}$ (depth); $0.00-1.20 \mathrm{ml} / \mathrm{L}, 1.20-2.00 \mathrm{ml} / \mathrm{L}$ and $2.00-2.40 \mathrm{ml} / \mathrm{L}$ (oxygen); $2.0-2.5^{\circ} \mathrm{C}, 2.5-4.0^{\circ} \mathrm{C}, 4.0-6.0^{\circ} \mathrm{C}$ and $6-6.5^{\circ} \mathrm{C}$ (temperature); $8-16 \%, 16 \%-18 \%$ and $18-20 \%$ (organic matter), 20-30\%, 60-70\% and 80-100\% (mud). Polychaetes were not distributed according to gradients of any of these environmental variables. The dominant and frequent species of each group were not restricted to unique assemblages. Results of a canonical correspondence analysis indicated that depth, oxygen and temperature were the main environmental variables responsible for polychaete distribution. The dominance of muddy sediments with high organic matter content ( 8.26 to $18.78 \%$ ) throughout the study area reduces the importance of these parameters for polychaete distribution, since the dominant and frequent species belong to the detritivore families Ampharetidae, Maldanidae and Cirratulidae.
\end{abstract}

Keywords: Polychaeta, oxygen minimum zone, temperature, depth, organic matter, granulometry, faunal distribution.

RESUMEN: RELACIÓN ENTRE POLIQUETOS DE MAR PROFUNDO Y FACTORES AMBIENTALES EN EL SURESTE DEL GOLFO DE CALIFORNIA, MÉXICO. - Se recolectaron poliquetos de fondos blandos en el mar profundo del sureste del golfo de California a $732-2110 \mathrm{~m}$ de profundidad, durante 4 cruceros oceanográficos, en 2000 y 2001 . En total se recolectaron 84 taxa representando a 32 familias. Se analizaron las relaciones entre densidad, número de especies y diversidad con profundidad, oxígeno disuelto, temperatura, granulometría y contenido de materia orgánica en el sedimento. Los dendrogramas y gráficos MDS basados en la densidad de poliquetos permitieron el establecimiento de grupos faunísticos para cada variable ambiental: $700-1100 \mathrm{~m}, 1100-1600 \mathrm{~m}, 1900-2100 \mathrm{~m}$ y $2100-2200 \mathrm{~m}$ (profundidad); $0.00-1.20 \mathrm{ml} / \mathrm{L}, 1.20-2.00 \mathrm{ml} / \mathrm{L}$ y $2.00-2.40$ $\mathrm{ml} / \mathrm{L}$ (oxígeno); $2.0-2.5^{\circ} \mathrm{C}, 2.5-4.0^{\circ} \mathrm{C}, 4.0-6.0^{\circ} \mathrm{C}$ y $6-6.5^{\circ} \mathrm{C}$ (temperatura); $8-16 \%, 16 \%-18 \%$ y $18-20 \%$ (materia orgánica), $20-30 \%, 60-70 \%$ y $80-100 \%$ (porcentaje de lodo). Los poliquetos no se distribuyeron de acuerdo con gradientes de ninguna de las variables ambientales estudiadas. Las especies dominantes y frecuentes de cada grupo no se restringieron a un solo grupo faunístico. Los resultados de un análisis de correspondencias canónicas indicaron que la profundidad, oxígeno disuelto y la temperatura fueron las principales variables ambientales responsables de la distribución de poliquetos. La dominancia de sedimentos lodosos con altos contenidos de materia orgánica (8.26 a 18.78\%) en el área de estudio reduce la importancia de estas variables en la distribución de poliquetos, ya que las especies dominantes y frecuentes pertenecen a las familias detritívoras Ampharetidae, Maldanidae y Cirratulidae.

Palabras clave: Polychaeta, zona de mínimo oxígeno, temperatura, profundidad, materia orgánica, granulometría, distribución faunística. 


\section{INTRODUCTION}

The southern Gulf of California connects with the Pacific Ocean at its mouth, where there are depths greater than $3000 \mathrm{~m}$, while the northern part is relatively shallow (Parker, 1964; Alvarez-Borrego and Schwartzlose, 1979). In the southern Gulf, water temperature decreases gradually with depth to values lower than $10^{\circ} \mathrm{C}$ at $400-500 \mathrm{~m}$ and $<4^{\circ} \mathrm{C}$ at $2000 \mathrm{~m}$. The southernmost basin, from about $26^{\circ} \mathrm{N}$ to the mouth of the Gulf, has epibenthic water temperatures of $2^{\circ} \mathrm{C}$ or less (Parker, 1964). Recently, Hendrickx (2003a) reported temperatures ranging from $2^{\circ} \mathrm{C}$ (at about $2000 \mathrm{~m}$ depth) to $9^{\circ} \mathrm{C}$ (at $550 \mathrm{~m}$ ) in the southeastern Gulf of California.

In the North Atlantic, the eastern Pacific and the Indian Ocean, organic carbon, bottom water oxygen concentration and depth strongly influence deepwater macrofaunal diversity. On the other hand, percentages of sand and clay have no significant effects on macrofaunal distribution in these zones (Levin and Gage, 1998). The importance of organic matter content in sediments has been established by Cook et al. (2000), Gooday et al. (2000) and Gooday et al. (2001), who observed that annelids often display parallel trends along oxygen and organic matter content gradients. Effects of depth have been reported in the North Atlantic, where a mid-slope diversity maximum is found following a unimodal pattern with a peak at intermediate depths, and lower diversity occurs at upper bathyal and abyssal depths. This pattern, however, does not appear to be universal (Levin et al., 2001).

Bottom water dissolved oxygen concentration is one of the major factors affecting the presence of benthic and demersal species of most marine invertebrates where hypoxia occurs (Rosenberg et al., 1991; Diaz and Rosenberg, 1995; Wishner et al., 1995; Levin and Gage, 1998; Levin et al., 2000; Levin, 2001). The oxygen minimum zones (OMZs) are midwater regions of the ocean with hypoxic waters, where oxygen concentrations are typically $<0.5 \mathrm{ml} / \mathrm{L}$, but vary in different regions of the world (Levin, 2001; 2003; Levin et al, 2001). Oxygen concentration has been shown to strongly influence distribution and diversity of benthic organisms (Wishner et al., 1995; Levin and Gage, 1998; Levin et al., 2000; Levin, 2001). This pattern has been reported on the West African margin (Sanders, 1969), in the eastern Pacific, in a seamount off Mexico (Levin et al., 1991), in the NW Arabian Sea off Oman (Levin et al., 1997, Levin et al., 2000) and on the Peru and Chile margins (Levin et al., 2002)

Parker (1964) recognised an OMZ in the southern and central Gulf of California with a $\leq 0.5 \mathrm{ml} / \mathrm{L}$ fringe that runs parallel to the coast on both sides of the Gulf to almost $29^{\circ} \mathrm{N}$. Recent studies performed in the southern Gulf of California evidenced a welloxygenated epipelagic zone from the surface to about 80-125 m, with oxygen concentrations as high as or higher than $2 \mathrm{ml} / \mathrm{L}$, and a wide zone of hypoxic to almost anoxic waters was found, extending roughly from $150-200 \mathrm{~m}$ to $600-800 \mathrm{~m}$ (Hendrickx, 2001). Oxygen gradually increases from $800 \mathrm{~m}$ and reaches hypoxic values below or close to $1 \mathrm{ml} / \mathrm{L}$ at $1500 \mathrm{~m}$, although values occasionally increase to 1.8 $\mathrm{m} / \mathrm{L}$ between 1400 and $1500 \mathrm{~m}$ (Hendrickx, 2003a). At depths greater than $2000 \mathrm{~m}$, there is a steady increase in the oxygen content, which progressively reaches values above $2 \mathrm{ml} / \mathrm{L}$ (Hendrickx, 2001).

Polychaetes are the most tolerant taxon to low oxygen (Diaz and Rosenberg, 1995) and are dominant in oxygen minimum zones (Levin, 2001; 2003). Polychaetes from deep-sea waters in the Gulf of California have been studied by Blake (1985), SolísWeiss and Hilbig (1992) and Solís-Weiss and Hernández-Alcántara (1994) in the hydrothermal vents from the Guaymas basin. A list of deep-water polychaetes based on samples from the southeastern Gulf of California was established by Méndez (2006). It shows that the deep-sea polychaete fauna from the Gulf of California is similar to that registered in adjacent areas by several authors such as Hartman $(1960,1963)$, in deep basins off southern California, and Fauchald (1972), who identified 227 polychaete species off the Mexican west coast and adjacent areas in the eastern Pacific Ocean. Other recent studies concerning deep-sea polychaetes in the northern Pacific correspond to Fauchald and Hanckock (1981) in Central Oregon and Blake and Hilbig (1990) in the Juan de Fuca and Explorer Ridge systems in the northeastern Pacific. Moreover, Blake and Hilbig (1994) and Blake et al. $(1995,1996,2000)$ performed a taxonomic atlas of polychaetes from the Santa Barbara Basin and western Santa Barbara Channel.

Studies of the environmental parameters influencing the distribution of deep-sea benthic fauna in the eastern Pacific are scarce. The US Environmental Protection Agency and the US Navy supported three studies (SAIC, 1991; 1992; Blake et al., 1992) on benthic infauna from the continental 


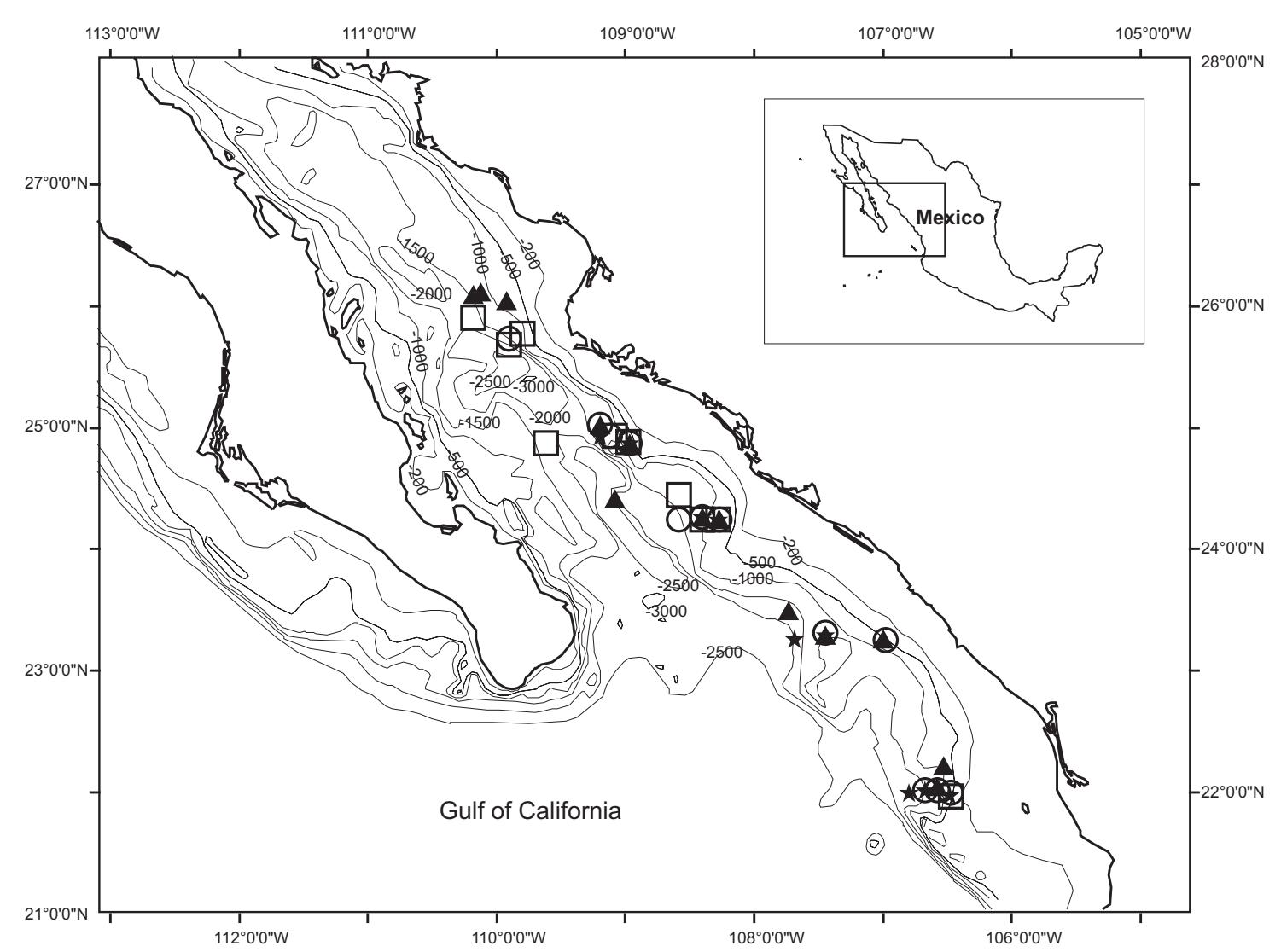

FIG. 1. - Study area showing the location of stations (TALUD $4=$ squares; TALUD $5=$ asterisks; TALUD $6=$ circles; TALUD $7=$ triangles).

slope off Farallon Islands near San Francisco. Levin and Gage (1998) and Levin et al. (1991) studied the relationships of environmental factors with macrobenthic communities in southern California and off Acapulco, Mexico, respectively. Wishner et al. (1995) studied the distribution of pelagic and benthic fauna in relation to the lower boundary of the oxygen minimum zone on a seamount off Acapulco. However, the analyses of the relationships between environmental parameters and deep-water polychaetes in the Gulf of California are non-existent. Recently, Hernández-Alcántara and Solís-Weiss (2005) related some environmental parameters to the Spionida from the Gulf of California, but their data correspond to the sublittoral zone, between depths of 22.2 and $120 \mathrm{~m}$.

The present paper examines the relationships between some environmental variables (depth, oxygen concentration, temperature, organic matter content in sediments and percentage of mud) and deepwater polychaete communities occurring at depths ranging from 732 to $2110 \mathrm{~m}$ in the southeast Gulf of California. Comparisons with previous studies in other regions of the world are also presented here.

\section{MATERIAL AND METHODS}

\section{Sampling and laboratory work}

The TALUD project, organised by the Laboratory of Invertebrates of the Mazatlán Marine Station (Instituto de Ciencias del Mar y Limnología, Universidad Nacional Autónoma de México, UNAM), is a multidisciplinary project encompassing the study of meiofauna, polychaetes, molluscs, crustaceans and fish on the continental slope of the southern Gulf of California. Deep-water polychaetes were collected aboard the R/V "El Puma" (UNAM) during four oceanographic cruises: TALUD 4 in August 2000 (11 stations), TALUD 5 during December 2000 (10 stations), TALUD 6 in March 2001 (12 stations), and TALUD 7 in June 2001 (13 stations) in the southeastern Gulf of California (Fig. 1).

Sediments to study macrofauna were collected with a $110 \mathrm{~cm}$ high $\mathrm{x} 40 \mathrm{~cm}$ wide $\mathrm{x} 20 \mathrm{~cm}$ thick (mean thickness) modified Karling dredge (Schlieper, 1972) towed for $30 \mathrm{~min}$. This dredge crawls in the sediment and can collect infauna and epifauna. It has two 7-cm wedges, which allow at most $85 \mathrm{~L}$ of sediment to be 
collected. Modifications include lateral stabilisers to prevent the dredge from turning over during deployment, and a removable rear plate (instead of a grid or sieve) allowing for easy collection of sediments after recovery of the gear. Since it is a heavy apparatus, it is assumed that it can collect the top $7 \mathrm{~cm}$ layer of sediment, but depending on the nature of the bottom, the collected volume may vary. Nevertheless, this allows quantitative studies based on the volume of sediment to be carried out. All the collected sediment was measured using graduated buckets and sieved through a $500 \mu \mathrm{m}$ mesh. The polychaetes were sorted and fixed with a $10 \%$ formaldehyde solution aboard the ship. Specimens found inside thick muddy tubes were fixed by adding $10 \%$ formaldehyde solution through one of the tube openings using a syringe. In the laboratory, the polychaetes were washed repeatedly and preserved in 70\% ethanol. They were counted and identified following the keys of Chamberlin (1919), Hartman (1944, 1957, 1960, 1968, 1969), Pettibone (1966), Fauchald (1968, 1970, 1972, 1977), Fauchald and Hancock (1981), Hernández-Alcántara (1992), Petersen (2000), Blake and Hilbig (1994) and Blake et al. $(1995,1996,2000)$.

Sediments (200-250 g) were taken from each sample to analyse grain size and organic matter content. Percentage of organic matter was obtained by loss of ignition at $550^{\circ} \mathrm{C}$ for $1 \mathrm{~h}$ (Dean, 1974). According to conventional methods (i.e. Folk, 1965), samples of about $200 \mathrm{~g}$ were sieved through a 2-mm mesh to separate gravel from sand and through a $0.0625-\mathrm{mm}$ mesh to separate sand from mud (the latter considered as a mixture of silt and clay). Grain size was expressed as percentages of mud $(<0.0625 \mathrm{~mm})$, sand $(0.0626-2.00 \mathrm{~mm})$ and gravel $(>2.01 \mathrm{~mm})$. Sampling depth was estimated with an analogical Edo Western echo sounder. Temperature was measured $c a .10 \mathrm{~m}$ above the bottom level with a CTD. Opening-closing bottles were used to obtain near-bottom water samples and oxygen concentrations were determined by the Winkler method (Strickland and Parsons, 1972). A Metrohm Herisau (Mod. E-415) burette was used, with an estimated precision of $2.34 \%$ and a lower limit of $0.01 \mathrm{ml} / \mathrm{L}$.

\section{Data analyses}

Abundance was expressed in terms of density of individuals/10 L of sediment, which is a quantitative measurement. The volume of collected sediment varied from 1.5 to $85 \mathrm{~L}$ throughout the study; thus, a standardisation was performed considering the volume of sediment collected at each station. The Shannon diversity index $\left(\mathrm{H}^{\prime} \log 2\right)$ was calculated with the Primer 5.1 for Windows program. Nonmetric multidimensional scaling (MDS) ordination plots (Bray-Curtis similarity index and fourth root of densities) were made with the data obtained on each cruise and with the total data obtained on the four cruises (Primer 5.1 for Windows) and showed that most of the samples were aggregated (stress $=0.01$ ). This rules out the seasonal and spatial influence on faunal distribution and indicates that other abiotic variables are responsible for polychaete distribution. The Pearson correlation coefficients (Excel 2000) were calculated between each pair of biotic (density, species number and diversity) and abiotic (depth, temperature, dissolved oxygen, organic matter and percentages of mud, sand and gravel) variables.

Faunal assemblages were determined according to depth, oxygen, temperature, organic matter content and percentage of mud by means of cluster analyses using species densities. Since no significant seasonal and spatial variability was detected, samples obtained during the entire study were pooled for depth ranges (each $100 \mathrm{~m}$ ), oxygen concentration ranges (each $0.4 \mathrm{ml} / \mathrm{L}$ ), temperature ranges (each $0.5^{\circ} \mathrm{C}$ ), organic matter content ranges (each $2 \%$ ) and percentage of mud ranges (each $10 \%$ ). MDS ordination plots were simultaneously built for each abiotic variable using the same ranges. Both dendrograms (group average link) and MDS ordination plots were built using the Primer 5.1 for Windows program following the Bray-Curtis similarity index, using the species density transformed to the fourth root, which downweights the effect of rare or abundant species (Field et al., 1982). The characteristic species of each assemblage were obtained through dominance and frequency calculated separately for each of the groups obtained in the cluster analyses. Dominant species $(\mathrm{Dm} \geq 1 \%)$ were determined according to the mean dominance index proposed by Picard $(1965)$, as $D m=\left(n_{i} / N\right) x 100$, where $\mathrm{Dm}=$ mean dominance index for species $\mathrm{i} ; \mathrm{n}_{\mathrm{i}}=$ number of individuals belonging to species $\mathrm{i}$; $\mathrm{N}=$ total number of individuals belonging to all the species. Following Glémarec (1964), frequency was calculated as $\mathrm{F}=\left(\mathrm{m}_{\mathrm{i}} \times 100\right) / \mathrm{M}$, where $\mathrm{m}_{\mathrm{i}}=$ number of samples in which species i appeared and $M=$ total number of samples. Frequent species had $\mathrm{F} \geq 15 \%$.

In order to closely determine the main factors influencing polychaete distribution in the study area, 
a canonical correspondence analysis (CCA) was performed (MVSP program, ver. 3.1, 1985-2002) considering the density of the dominant and frequent species (throughout the study, considering the 44 samples) as biotic variables and depth, temperature, dissolved oxygen, organic matter content and percentages of mud, sand and gravel as abiotic variables. Depth, dissolved oxygen and temperature ranges were plotted for the dominant and frequent species in the study. Only species identified to specific and generic levels were included in these plots.

\section{RESULTS}

Forty-four stations were sampled throughout the study, of which only two (Station 3, Talud 4 and Station 12, Talud 6) did not contain polychaetes. A total of 84

TABLE 1. - List of species collected during the study.

\section{Orbiniidae}

Califia calida Hartman, 1957

Leitoscoloplos elongatus (Johnson, 1901)

Phylo nudus (Moore, 1911)

Paraonidae

Aricidea alisetosa Fauchald, 1972

Aricidea longicirrata Fauchald, 1972

Paraonis pycnobranchiata Fauchald, 1972

Spionidae

Polydora sp

Prionospio vermillionensis Fauchald, 1972

Spiophanes fimbriata Moore, 1923

Poecilochaetidae

Poecilochaetus johnsoni Hartman, 1939

Heterospionidae

Heterospio catalinensis (Hartman, 1944)

Chaetopteridae

Phyllochaetopterus limicolus Hartman, 1960

Cirratulidae

Aphelochaeta monilaris Hartman, 1960

Aphelochaeta multifilis (Moore, 1909)

Chaetozone corona Berkeley and Berkeley, 1941

Chaetozone setosa Malmgren, 1867

Cirratulus sp

Monticellina tesselata (Hartman, 1960)

Tharyx sp

Capitellidae

Neoheteromastus lineus Hartman, 1960

Notomastus sp

Maldanidae

Asychis ramosus Levenstein, 1961

Maldane cristata Treadwell, 1923

Maldanidae sp 1

Maldanidae sp 2

Maldanidae sp 3

Maldanidae sp 4

Maldanidae sp 5

Maldanidae sp 6

Maldanidae sp 7

Maldanidae sp 8

Maldanidae indeterminable

Opheliidae

Ophelina acuminata (Hartman, 1960)

Travisia brevis Moore, 1923

Opheliidae indeterminable

Scalibregmatidae

Scalibregma inflatum Rathke, 1843

Phyllodocidae

Eteone sp

Eulalia anoculata Fauchald, 1972

Eulalia strigata Hartman, 1968

Paranaitis polynoides (Moore, 1909)

Polynoidae

Halosydna johnsoni (Darboux, 1899)

Polynoidae indeterminable

Sigalionidae

Leanira alba Moore, 1910

Sthenolepis sp
Pilargiidae

Ancistrosyllis hartmanae Pettibone, 1966

Sigambra sp

Syllidae

Exogone lourei Berkeley and Berkeley, 1938

Nereididae

Ceratocephale pacifica Hartman, 1960

Ceratonereis vermillionensis Fauchald, 1972

Nereis anoculopsis Fauchald, 1972

Nereididae indeterminable

Glyceridae

Glyceridae indeterminable

Goniadidae

Goniada brunnea Treadwell, 1906

Nephtyidae

Nephtys paradoxa Malm, 1874

Amphinomidae

"Pareurythoe" sp

Pseudeurythoe ambigua (Monro, 1933)

Onuphidae

Onuphis iridescens (Johnson, 1901)

Onuphis profundi Fauchald, 1968

Eunicidae

Eunice megabranchia Fauchald, 1970

Lumbrineridae

Eranno bicirrata (Treadwell, 1929)

Lumbrineris cedroensis Fauchald, 1970

Ninoe foliosa Fauchald, 1972

Ninoe fuscoides Fauchald, 1972

Ninoe longibranchia Fauchald, 1972

Lumbrineridae indeterminable

Arabellidae

Drilonereis falcata Moore, 1911

Sternaspidae

Sternaspis fossor Stimpson, 1854

Flabelligeridae

Brada pluribranchiata (Moore, 1923)

Ilyphagus ilyvestis Hartman 1960

Pherusa abyssalis Fauchald, 1972

Fauveliopsidae

Fauveliopsis rugosa Fauchald, 1972

Ampharetidae

Ampharete arctica Malmgren, 1866

Melinna exilia Fauchald, 1972

Melinnampharete eoa Annenkova, 1937

Mexamage corrugata Fauchald, 1972

Samythella pala Fauchald, 1972

Schistocomus hiltoni Chamberlin, 1919

Ampharetidae indeterminable

Terebellidae

Thelepus hamatus Moore, 1905

Thelepus setosus (Quatrefages, 1865)

Streblosoma crassibranchia Treadwell, 1914

Trichobranchidae

Terebellides stroemi Sars, 1835

Sabellidae

Chone gracilis Moore, 1906

Sabellidae indeterminable 
species, representing 32 families, were collected, from which 61 taxa were identified to species level (Table 1). Species belonging to the genera Polydora, Cirratulus, Tharyx, Notomastus, Eteone, Sthenolepis, Sigambra and "Pareurythoe" were not identified to species due to the poor condition of the specimens. Important taxonomic structures from several specimens from the families Maldanidae, Opheliidae, Polynoidae, Nereididae, Glyceridae, Lumbrineridae, Ampharetidae and Sabellidae had been damaged, making further identification impossible. These specimens were catalogued as "indeterminable" within their respective families. In some cases, species names used in Méndez (2006) were updated due to recent changes in nomenclature cited in the literature.
The families best represented in the study area in terms of number of species were Maldanidae (11 taxa, of which 9 were not identified to species), Cirratulidae and Ampharetidae with 7 species each, and Lumbrineridae, with 6 species. The 26 dominant $(\mathrm{Dm} \geq 1 \%)$ and frequent $(\mathrm{F} \geq 15 \%)$ species in the study are listed in the Figure 7 caption. The highest mean dominance values (14 to 5\%) corresponded to Monticellina tesselata, Fauveliopsis rugosa, Maldanidae sp 1, Neoheteromastus lineus and Onuphis iridescens. The highest frequency values (32 to 25\%) corresponded to Melinna exilia, Phyllochaetopterus limicolus, Califia calida, Maldanidae indeterminable and Onuphis iridescens. The families best represented by dominant and fre-

TABLE 2. - Latitude, longitude, mean depth, temperature, dissolved oxygen, organic matter content, percentages of mud $(<0.0625 \mathrm{~mm})$, sand $(0.0626-2.00 \mathrm{~mm})$ and gravel $(>2.01 \mathrm{~mm})$, total density, species number and diversity recorded over the study.

\begin{tabular}{|c|c|c|c|c|c|c|c|c|c|c|c|c|c|}
\hline Talud & Station & $\begin{array}{l}\text { Latitude } \\
(\mathrm{N})\end{array}$ & $\begin{array}{l}\text { Longitude } \\
\text { (W) }\end{array}$ & $\begin{array}{c}\text { Mean } \\
\text { depth (m) }\end{array}$ & $\begin{array}{c}\text { Temp } \\
\left({ }^{\circ} \mathrm{C}\right)\end{array}$ & $\begin{array}{c}\text { Oxygen } \\
(\mathrm{ml} / \mathrm{L})\end{array}$ & $\begin{array}{c}\text { Organic } \\
\text { matter }(\%)\end{array}$ & $\underset{(\%)}{\operatorname{Mud}}$ & $\begin{array}{c}\text { Sand } \\
(\%)\end{array}$ & $\begin{array}{c}\text { Gravel } \\
(\%)\end{array}$ & $\begin{array}{l}\text { Total density } \\
\text { (Ind/10L) }\end{array}$ & $\begin{array}{l}\text { Species } \\
\text { number }\end{array}$ & $\begin{array}{l}\text { Diversity } \\
\text { (H'log2) }\end{array}$ \\
\hline \multirow[t]{10}{*}{4} & 3 & $21^{\circ} 58^{\prime}$ & $106^{\circ} 28^{\prime}$ & 793 & 3.6 & 0.19 & 18.78 & 99.4 & 0.6 & 0.0 & 0.000 & 0 & 0.000 \\
\hline & 18 & $24^{\circ} 15^{\prime}$ & $108^{\circ} 17^{\prime}$ & 926 & & 1.03 & 14.03 & 99.2 & 0.8 & 0.0 & 3.827 & 7 & 2.261 \\
\hline & 19 & $24^{\circ} 15^{\prime}$ & $108^{\circ} 24^{\prime}$ & 1198 & 3.7 & 0.73 & 15.19 & 99.5 & 0.5 & 0.0 & 1.190 & 7 & 2.721 \\
\hline & 20 & $24^{\circ} 27^{\prime}$ & $108^{\circ} 35^{\prime}$ & 1510 & 3.1 & 1.26 & 15.47 & 98.1 & 1.9 & 0.0 & 1.666 & 9 & 3.042 \\
\hline & 25 & $24^{\circ} 53^{\prime}$ & $108^{\circ} 59^{\prime}$ & 789 & 5.1 & 0.29 & 9.86 & 99.1 & 0.9 & 0.0 & 3.926 & 10 & 2.693 \\
\hline & 26 & $24^{\circ} 56^{\prime}$ & $109^{\circ} 05^{\prime}$ & 1237 & 3.7 & 0.76 & 14.69 & 99.4 & 0.6 & 0.0 & 1.547 & 9 & 3.084 \\
\hline & 28 & $24^{\circ} 52^{\prime}$ & $109^{\circ} 37^{\prime}$ & 2032 & 2.0 & 2.20 & 14.96 & 99.2 & 0.8 & 0.0 & 3.078 & 7 & 2.870 \\
\hline & 33 & $25^{\circ} 46^{\prime}$ & $109^{\circ} 48^{\prime}$ & 1075 & 4.3 & 0.51 & 13.86 & 99.9 & 0.1 & 0.0 & 6.381 & 12 & 2.701 \\
\hline & 34 & $25^{\circ} 41^{\prime}$ & $109^{\circ} 54^{\prime}$ & 1220 & 3.5 & 0.79 & 8.26 & 20.6 & 79.4 & 0.0 & 26.006 & 1 & 0.000 \\
\hline & 35 & $25^{\circ} 54^{\prime}$ & $110^{\circ} 11^{\prime}$ & 2050 & 2.5 & 1.68 & 10.27 & 93.5 & 6.5 & 0.0 & 0.899 & 4 & 2.000 \\
\hline \multirow{9}{*}{5} & 3 & $21^{\circ} 59^{\prime}$ & $106^{\circ} 29^{\prime}$ & 732 & 5.5 & 0.12 & 17.23 & 100.0 & 0.0 & 0.0 & 0.303 & 1 & 0.000 \\
\hline & 5 & $22^{\circ} 01^{\prime}$ & $106^{\circ} 40^{\prime}$ & 1568 & 3.0 & 1.15 & 15.67 & 99.9 & 0.1 & 0.0 & 1.200 & 6 & 2.417 \\
\hline & 6 & $22^{\circ} 00^{\prime}$ & $106^{\circ} 48^{\prime}$ & 1980 & 2.2 & 1.88 & 15.50 & 94.9 & 5.1 & 0.0 & 0.758 & 5 & 2.322 \\
\hline & 12 & $23^{\circ} 18^{\prime}$ & $107^{\circ} 27^{\prime}$ & 1165 & 3.8 & 0.54 & 11.03 & 26.5 & 73.5 & 0.0 & 1.905 & 4 & 2.000 \\
\hline & 14 & $23^{\circ} 16^{\prime}$ & $107^{\circ} 41^{\prime}$ & 2110 & 2.1 & 1.98 & 13.64 & 100.0 & 0.0 & 0.0 & 0.741 & 4 & 2.000 \\
\hline & 18 & $24^{\circ} 15^{\prime}$ & $108^{\circ} 17^{\prime}$ & 965 & 5.0 & 0.15 & 13.74 & 100.0 & 0.0 & 0.0 & 3.077 & 2 & 0.811 \\
\hline & 19 & $24^{\circ} 16^{\prime}$ & $108^{\circ} 24^{\prime}$ & 1190 & 4.0 & 0.38 & 14.05 & 100.0 & 0.0 & 0.0 & 2.400 & 5 & 1.922 \\
\hline & 25 & $24^{\circ} 52^{\prime}$ & $108^{\circ} 58^{\prime}$ & 830 & 5.2 & 0.13 & 10.87 & 98.1 & 1.5 & 0.4 & 8.667 & 4 & 1.352 \\
\hline & 26 & $24^{\circ} 56^{\prime}$ & $109^{\circ} 12^{\prime}$ & 1295 & 3.8 & 0.53 & 13.81 & 100.0 & 0.0 & 0.0 & 0.946 & 6 & 2.523 \\
\hline \multirow[t]{12}{*}{6} & 3 & $22^{\circ} 00^{\prime}$ & $106^{\circ} 28^{\prime}$ & 775 & 5.8 & 0.21 & 19.90 & 98.8 & 1.2 & 0.0 & 2.406 & 2 & 1.000 \\
\hline & 4 & $22^{\circ} 01^{\prime}$ & $106^{\circ} 35^{\prime}$ & 1220 & 3.7 & & 14.67 & 91.8 & 8.2 & 0.0 & 7.218 & 3 & 1.000 \\
\hline & 5 & $22^{\circ} 01^{\prime}$ & $106^{\circ} 40^{\prime}$ & 1500 & 2.8 & 1.74 & 13.36 & 92.7 & 7.3 & 0.0 & 4.511 & 10 & 3.191 \\
\hline & 11 & $23^{\circ} 15^{\prime}$ & $106^{\circ} 59^{\prime}$ & 840 & 5.9 & 0.17 & 15.58 & 98.8 & 1.2 & 0.0 & 0.642 & 1 & 0.000 \\
\hline & 12 & $23^{\circ} 19^{\prime}$ & $107^{\circ} 27^{\prime}$ & 1105 & 4.1 & 0.50 & 10.66 & 93.6 & 6.4 & 0.0 & 0.000 & 0 & 0.000 \\
\hline & 18 & $24^{\circ} 15^{\prime}$ & $108^{\circ} 16^{\prime}$ & 920 & 5.3 & 0.29 & 12.21 & 99.8 & 0.2 & 0.0 & 4.942 & 7 & 2.034 \\
\hline & 19 & $24^{\circ} 16^{\prime}$ & $108^{\circ} 24^{\prime}$ & 1180 & 3.7 & 0.73 & 14.41 & 99.6 & 0.4 & 0.0 & 4.346 & 6 & 2.522 \\
\hline & 20 & $24^{\circ} 15^{\prime}$ & $108^{\circ} 35^{\prime}$ & 1345 & 3.0 & 1.28 & 14.52 & 99.7 & 0.3 & 0.0 & 9.828 & 13 & 3.490 \\
\hline & 25 & $24^{\circ} 52^{\prime}$ & $108^{\circ} 58^{\prime}$ & 840 & 5.6 & 0.22 & 10.90 & 97.3 & 2.7 & 0.0 & 3.574 & 7 & 2.603 \\
\hline & 26 & $24^{\circ} 56^{\prime}$ & $109^{\circ} 07^{\prime}$ & 1230 & 3.7 & 1.40 & 13.06 & 99.5 & 0.5 & 0.0 & 19.247 & 6 & 2.322 \\
\hline & 27 & $25^{\circ} 01^{\prime}$ & $109^{\circ} 12^{\prime}$ & 1590 & 2.8 & 1.43 & 11.58 & 97.8 & 2.2 & 0.0 & 20.379 & 11 & 3.197 \\
\hline & 34 & $25^{\circ} 44^{\prime}$ & $109^{\circ} 54^{\prime}$ & 1200 & 3.5 & 0.87 & 14.41 & 99.6 & 0.4 & 0.0 & 4.249 & 11 & 3.341 \\
\hline \multirow[t]{13}{*}{7} & 3 & $22^{\circ} 13^{\prime}$ & $106^{\circ} 32^{\prime}$ & 745 & 6.2 & 0.77 & 17.42 & 60.3 & 1.0 & 38.7 & 1.445 & 1 & 0.000 \\
\hline & 4 & $22^{\circ} 03^{\prime}$ & $106^{\circ} 35^{\prime}$ & 1215 & 3.6 & 0.82 & 8.55 & 93.7 & 4.6 & 1.7 & 5.245 & 13 & 2.534 \\
\hline & 11 & $23^{\circ} 16^{\prime}$ & $107^{\circ} 00^{\prime}$ & 785 & 5.6 & 0.15 & 17.47 & 100.0 & 0.0 & 0.0 & 3.727 & 1 & 0.000 \\
\hline & 12 & $23^{\circ} 18^{\prime}$ & $107^{\circ} 27^{\prime}$ & 1080 & 3.9 & 0.18 & 13.97 & 99.9 & 0.1 & 0.0 & 26.973 & 3 & 1.500 \\
\hline & $13 \mathrm{~B}$ & $23^{\circ} 30^{\prime}$ & $107^{\circ} 44^{\prime}$ & 1425 & 3.0 & 1.04 & 10.55 & 88.4 & 10.4 & 1.2 & 7.080 & 14 & 3.333 \\
\hline & 18 & $24^{\circ} 15^{\prime}$ & $108^{\circ} 16^{\prime}$ & 980 & 5.2 & 0.13 & 13.12 & 100.0 & 0.0 & 0.0 & 3.161 & 4 & 1.922 \\
\hline & 19 & $24^{\circ} 16^{\prime}$ & $108^{\circ} 24^{\prime}$ & 1170 & 3.6 & 0.08 & 12.56 & 100.0 & 0.0 & 0.0 & 3.372 & 8 & 2.922 \\
\hline & 25 & $24^{\circ} 52^{\prime}$ & $108^{\circ} 58^{\prime}$ & 815 & 4.7 & 0.04 & 12.21 & 99.7 & 0.3 & 0.0 & 35.402 & 4 & 0.949 \\
\hline & 26 & $24^{\circ} 25^{\prime}$ & $109^{\circ} 05^{\prime}$ & 1200 & 3.6 & 0.35 & 13.42 & 97.6 & 2.4 & 0.0 & 1.156 & 6 & 2.406 \\
\hline & 27 & $25^{\circ} 02^{\prime}$ & $109^{\circ} 12^{\prime}$ & 1550 & 2.7 & 1.39 & 14.76 & 100.0 & 0.0 & 0.0 & 13.824 & 15 & 3.021 \\
\hline & $32 \mathrm{~B}$ & $26^{\circ} 03^{\prime}$ & $109^{\circ} 55^{\prime}$ & 865 & 5.9 & 0.10 & 14.74 & 100.0 & 0.0 & 0.0 & 1.445 & 1 & 0.000 \\
\hline & $33 \mathrm{~B}$ & $26^{\circ} 07^{\prime}$ & $110^{\circ} 07^{\prime}$ & 1270 & 3.6 & 0.60 & 15.87 & 99.8 & 0.2 & 0.0 & 13.487 & 22 & 4.194 \\
\hline & $34 \mathrm{~B}$ & $26^{\circ} 06^{\prime}$ & $110^{\circ} 11^{\prime}$ & 1510 & 3.0 & 0.78 & 15.36 & 100.0 & 0.0 & 0.0 & 76.874 & 10 & 2.197 \\
\hline
\end{tabular}


TABLE 3. - Pearson correlation coefficients between abiotic and biotic variables $\left(* * * \mathrm{p}<0.001 ;{ }^{* *} \mathrm{p}<0.01 ;{ }^{*} \mathrm{p}<0.05\right.$; NS $=$ non significant $)$.

\begin{tabular}{llclllll}
\hline Depth & Temperature Oxygen & $\begin{array}{c}\text { Organic } \\
\text { matter }\end{array}$ & $\%$ Mud & $\%$ Sand & $\%$ Gravel & Density & $\begin{array}{c}\text { Species } \\
\text { number }\end{array}$ \\
\hline
\end{tabular}

\begin{tabular}{|c|c|c|}
\hline Depth & 1.000 & \\
\hline Temperature & $* * *-0.890$ & 1.000 \\
\hline Oxygen & $* * * 0.873$ & $* * *-0.799$ \\
\hline Organic matter & $*-0.102$ & $* * 0.167$ \\
\hline$\%$ Mud & NS & NS \\
\hline$\%$ Sand & NS & $*-0.107$ \\
\hline$\%$ Gravel & $* * *-0.203$ & $* * * 0.301$ \\
\hline Density & NS & $* *-0.160$ \\
\hline Species number & $* * * 0.297$ & $* * *-0.441$ \\
\hline Diversity & $* * * 0.476$ & $* * *-0.594$ \\
\hline
\end{tabular}

\begin{tabular}{l}
1.000 \\
$\mathrm{NS}$ \\
$\mathrm{NS}$ \\
$\mathrm{NS}$ \\
$\mathrm{NS}$ \\
$\mathrm{NS}$ \\
$* * * 0.327$ \\
$* * * 0.472$ \\
\hline
\end{tabular}

$$
\begin{aligned}
& 1.000 \\
& \begin{array}{rr}
* * * 0.331 & 1.000 \\
* * *-0.423 & * * *-0.938
\end{array} \\
& * * *-0.423 * * * *_{-}-0.938 \quad 1.000 \\
& * * 0.204 * * *-0.311 \\
& \text { NS NS } \\
& \text { ** }-0.168 \quad * * 0.219 \quad * *-0.170 \quad * *-0.186
\end{aligned}
$$$$
\text { NS NS }
$$

*** $0.253 \quad * *-0.172 * * *-0.256 \quad$ NS $\quad * * * 0.870 \quad 1.000$

quent species were Ampharetidae (5 species), Cirratulidae and Maldanidae (4 species each).

Station locations and their corresponding abiotic variables (depth, temperature, dissolved oxygen, organic matter content of sediments and percentages of mud, sand and gravel) and biotic variables (density, species number and diversity) are presented in Table 2. The Pearson correlation index indicated that depth, temperature and oxygen were strongly correlated among themselves and were the main abiotic variables influencing species number and diversity. Density showed a significant negative correlation with temperature. Organic matter in sediments also had a significant correlation, albeit lower, with species number and diversity. Percentages of mud and gravel seemed to have a stronger correlation with diversity (Table 3 ).

The cluster analysis based on data pooled in $100 \mathrm{~m}$ depth ranges indicated four depth groups: 700-1100 m (including 17 samples), 1100-1600 m (23 samples), 1900-2100 m (3 samples), and 2100-2200 m (one sample) (Fig. 2a). An oxygen concentration range of $0.2 \mathrm{ml} / \mathrm{L}$ was considered initially, but the resulting interpretation was confusing since lower oxygen groups (i.e. $0-0.2 \mathrm{ml} / \mathrm{L}$ and $0.2-0.4 \mathrm{ml} / \mathrm{L}$ ) were grouped with the $1.2 \mathrm{mg} / \mathrm{L}$ group and intermediate groups resulted independent. On the other hand, when the grouping was made every $0.5 \mathrm{ml} / \mathrm{L}$, valuable information was lost, since samples from 0 to $1.5 \mathrm{ml} / \mathrm{L}$ were grouped at a $50 \%$ similitude. A better fit using oxygen concentration values was obtained when a 0.4 $\mathrm{ml} / \mathrm{L}$ range was analysed. Three oxygen assemblages based on polychaete density were obtained (Fig. 3a): from 0 to $1.2 \mathrm{ml} / \mathrm{L}$ (33 samples), from 1.2 to $2.0 \mathrm{ml} / \mathrm{L}$ (nine samples) and from 2.0 to $2.4 \mathrm{ml} / \mathrm{L}$ (1 sample). Four faunal assemblages were established according to temperature (Fig. $4 \mathrm{a}$ ): from 2.0 to $2.5^{\circ} \mathrm{C}$ (three samples), from 2.5 to $4.0^{\circ} \mathrm{C}$ (24 samples), from 4.0 to $6.0^{\circ} \mathrm{C}$ (15 samples) and 6.0 to $6.5^{\circ} \mathrm{C}$ (one sample).
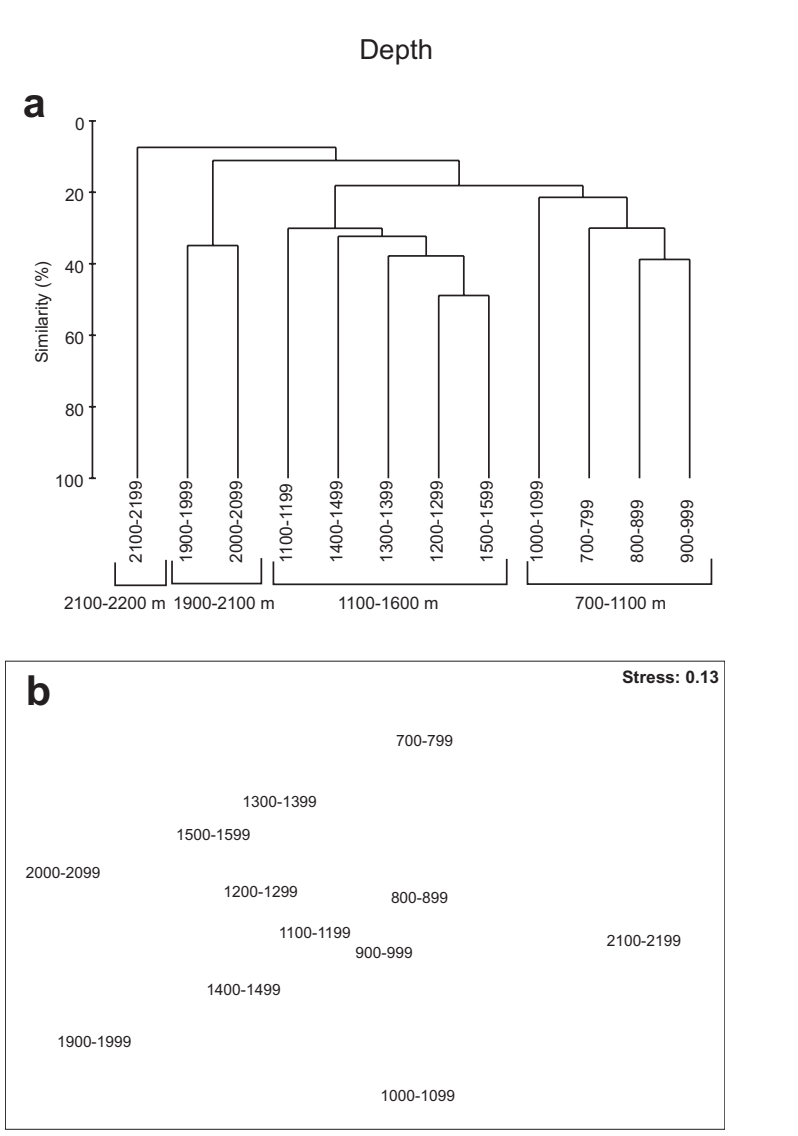

FIG. 2. - Depth ranges (grouped to the nearest $100 \mathrm{~m}$ ) according to polychaete densities. a) Dendrogram (Bray-Curtis similarity index; group average link); b) Non-metric multi-dimensional scaling ordination.

The majority of samples (39) corresponded to sediment with 8 to $16 \%$ organic matter content. Two independent groups were observed from 16 to $18 \%$ (three samples) and from 18 to 20\% (two samples) (Fig. 5a). Because most sediment samples were dominated by mud, the faunal analyses were performed using this component only. Three independent groups were obtained, of which only that corresponding to $80-100 \%$ of mud was representative, 
Dissolved oxygen
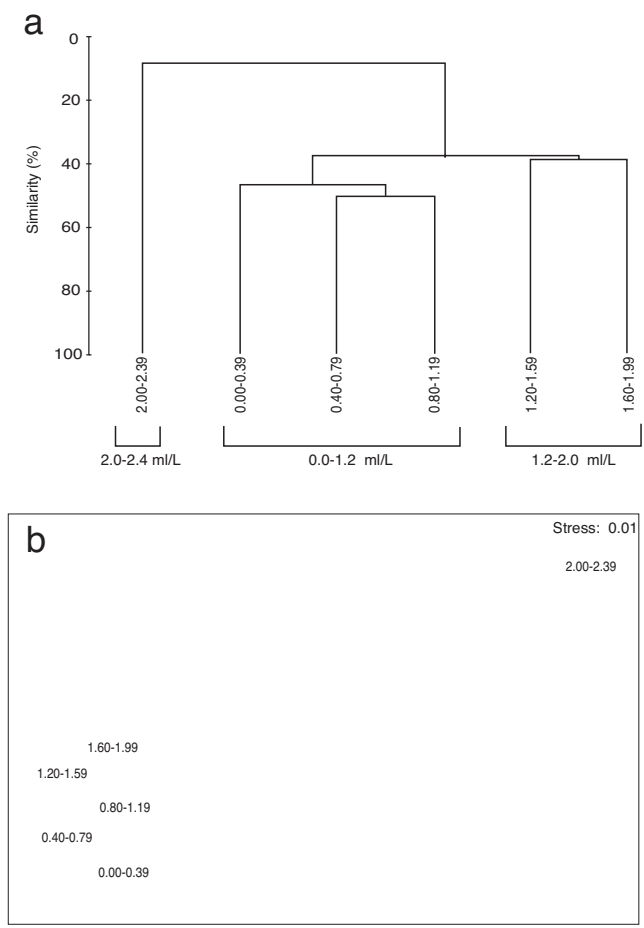

FIG. 3. - Dissolved oxygen ranges (grouped to the nearest $0.4 \mathrm{ml} / \mathrm{L}$ ), according to polychaete densities. a) Dendrogram (Bray-Curtis similarity index; group average link); b) non-metric multi-dimensional scaling ordination.
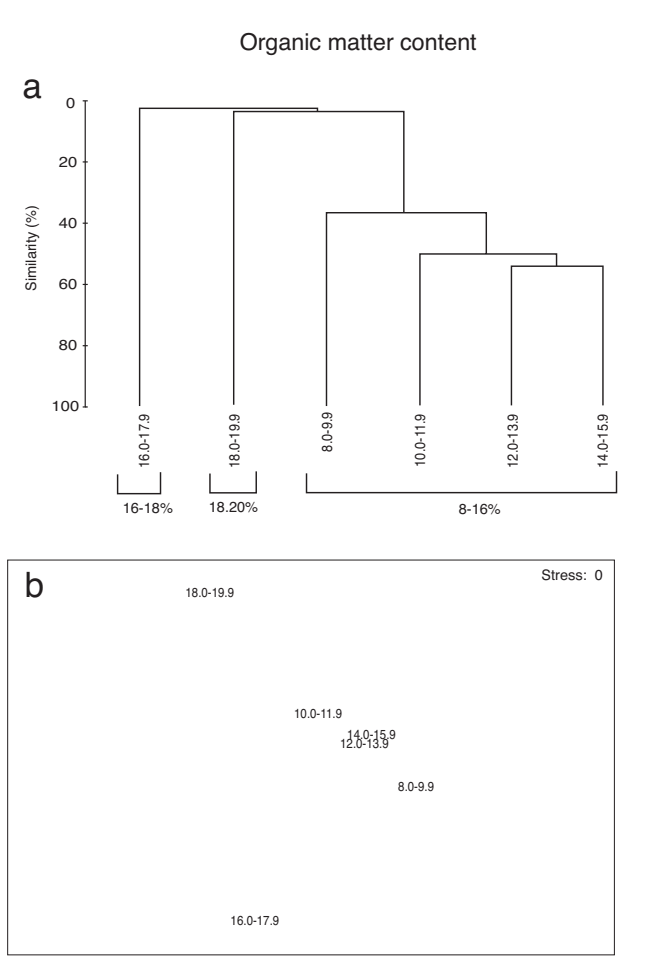

FIG. 5. - Organic matter content ranges (grouped to the nearest $2.0 \%$ ), according to polychaete densities. a) Dendrogram (BrayCurtis similarity index; group average link); b) non-metric multidimensional scaling ordination.
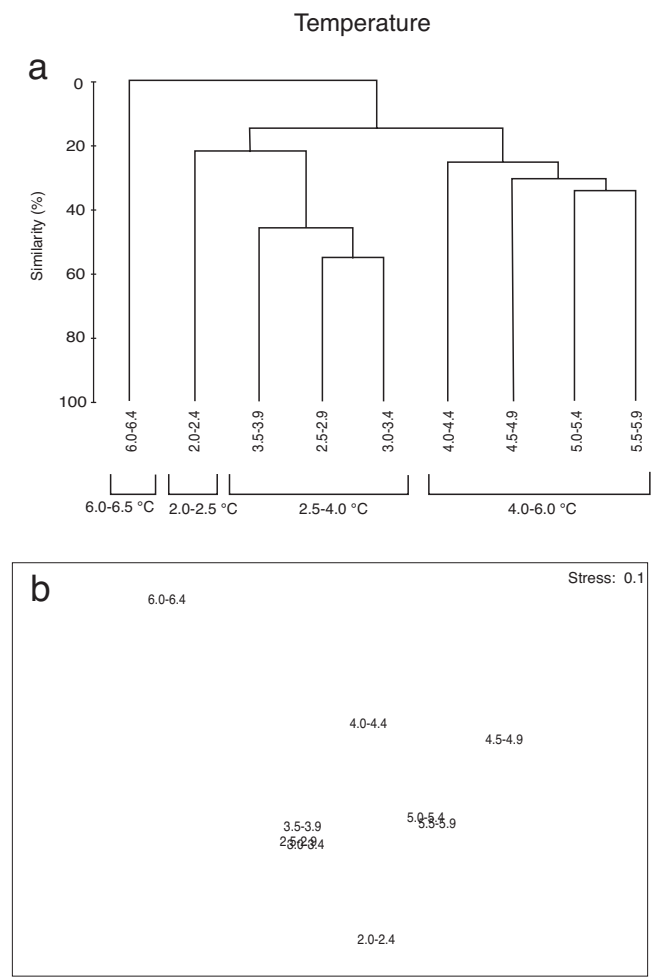

FIG. 4. - Temperature ranges (grouped to the nearest $0.5^{\circ} \mathrm{C}$ ), according to polychaete densities. a) Dendrogram (Bray-Curtis similarity index; group average link); b) non-metric multi-dimensional scaling ordination.
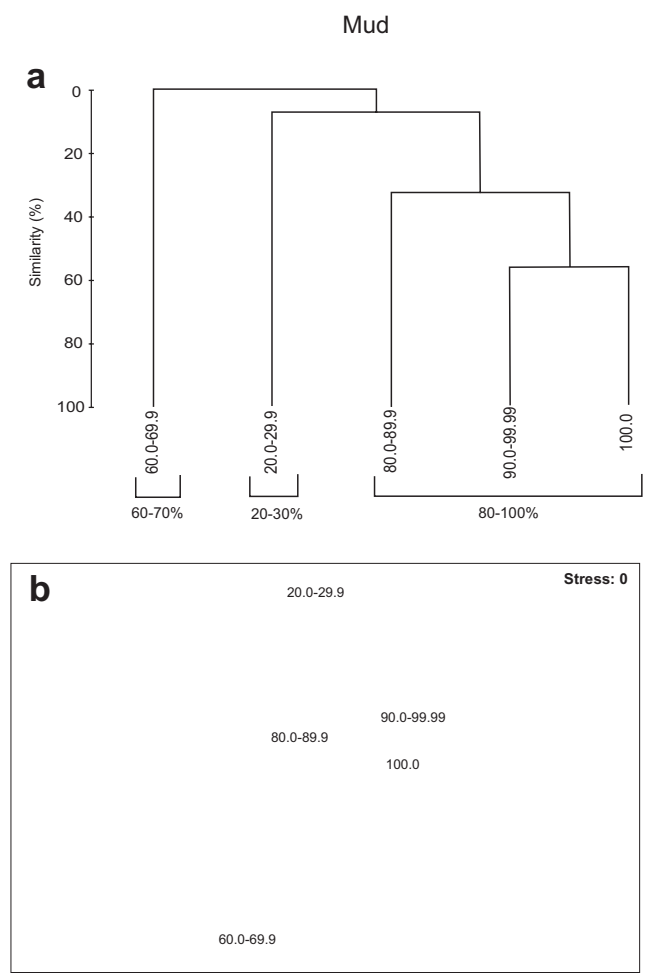

FIG. 6. - Percentage of mud ranges (grouped to the nearest 10\%), according to polychaete densities. a) Dendrogram (Bray-Curtis similarity index; group average link); b) non-metric multi-dimensional scaling ordination. 
since it included 42 samples. The other two groups (20-30\% and $60-70 \%)$ corresponded to only one sample each (Fig. 6a).

The MDS ordination plots carried out for each abiotic variable confirmed the groups obtained through the respective dendrograms; however, these graphs suggest that polychaete distribution did not show clear patterns following gradients of depth (Fig. 2b), of oxygen concentration (Fig. 3b), of temperature (Fig. 4b), of organic matter content (Fig. $5 b)$ or of mud percentage (Fig. 6b).

The ranges and mean values of the biotic and abiotic variables estimated for each faunal assemblage according to the analysed environmental variables are summarised in Tables 4 (depth), 5 (oxygen), 6 (temperature), 7 (organic matter content), and 8 (mud percentage). These tables also contain the characteristic species (dominant and frequent) of each assemblage. In most of the cases, several species were characteristic of more than one assemblage, which suggests wide distribution patterns (i.e. Califia calida, Maldane cristata, Phylochaetopterus limiculus and Melinna exilia). The grouping obtained for organic matter content and mud percentage was vast, since the majority of the samples were included in the same assemblage, thus making interpretation difficult.

The spatial distributions of the 26 dominant and frequent species in the study according to the biotic variables obtained in the CCA indicated that $34.6 \%$ of the variance is explained by the first canonical axis (eigenvalues $=0.688$ ) and $22.1 \%$ by the second canonical axis (eigenvalues $=0.438$ ). A high correlation was found between polychaete composition and the environmental variables on the first two axes (first axis 0.933; second axis 0.914). Depth was the most important environmental variable explaining species distribution, although this distribution was also strongly influenced by oxygen and temperature. Organic matter and especially granulometry were less important (Fig. 7).

Most of the species collected in this study were found over wide temperature, oxygen and depth ranges; however, the dominant and frequent species Aphelochaeta multifilis, Monticellina tesselata, Neoheteromastus lineus, Exogone lourei, Ceratocephale pacifica, Onuphis iridescens, Brada pluribranchiata, Fauveliopsis rugosa and Ampharete arctica were found within limited ranges of these variables (Fig. 8)

TABLE 4. - Ranges and mean values of the biotic and abiotic variables and characteristic species of each depth group.

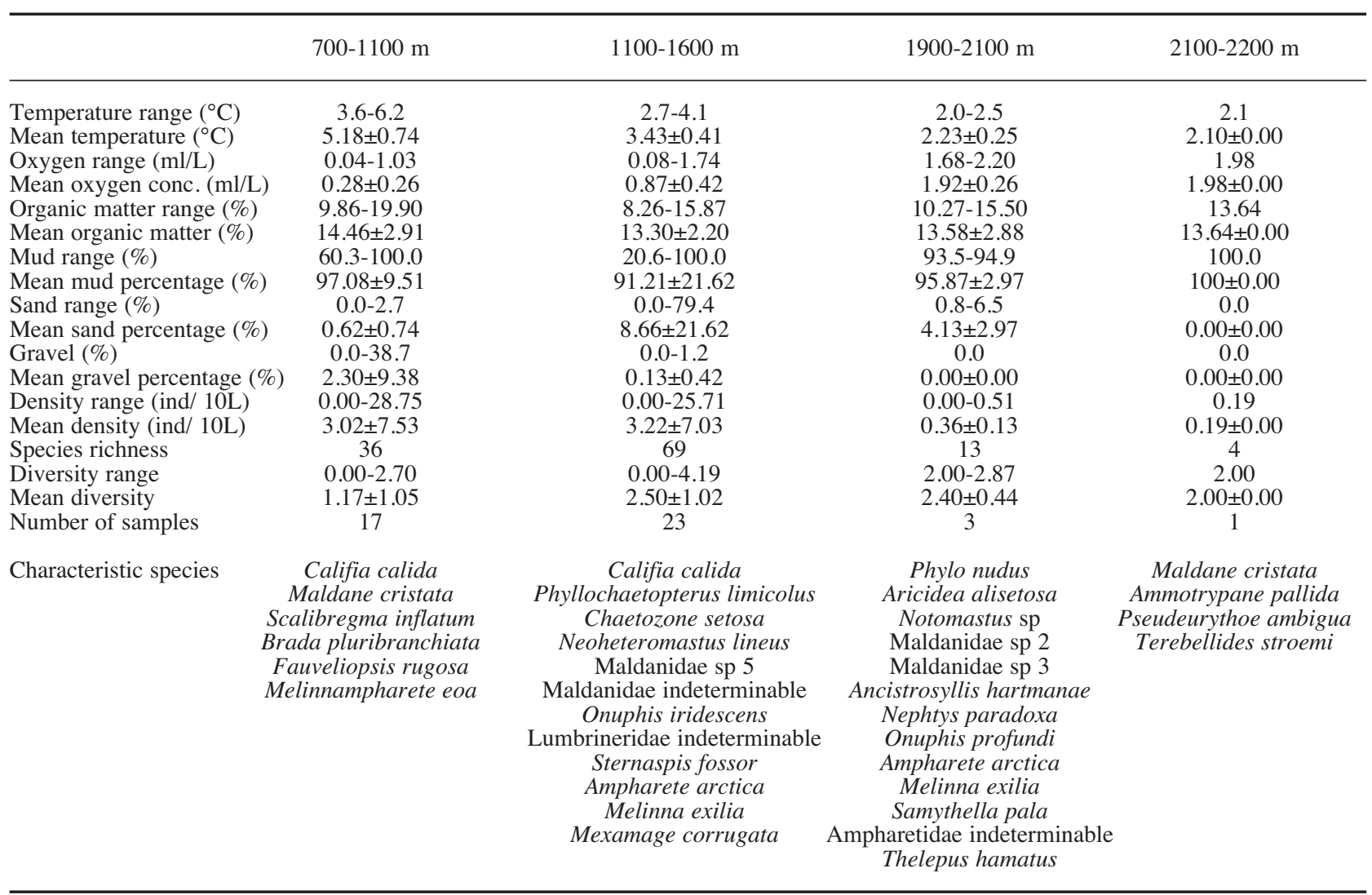


TABLE 5. - Ranges and mean values of the biotic and abiotic variables and characteristic species of each dissolved oxygen group.

\begin{tabular}{|c|c|c|c|}
\hline & $0.0-1.2 \mathrm{ml} / \mathrm{L}$ & $1.2-2.0 \mathrm{ml} / \mathrm{L}$ & $2.0-2.4 \mathrm{ml} / \mathrm{L}$ \\
\hline $\begin{array}{l}\text { Depth range }(\mathrm{m}) \\
\text { Mean depth }(\mathrm{m}) \\
\text { Temperature range }\left({ }^{\circ} \mathrm{C}\right) \\
\text { Mean temperature }\left({ }^{\circ} \mathrm{C}\right) \\
\text { Organic matter range }(\%) \\
\text { Mean organic matter }(\%) \\
\text { Mud range }(\%) \\
\text { Mean mud percentage }(\%) \\
\text { Sand range }(\%) \\
\text { Mean sand percentage }(\%) \\
\text { Gravel }(\%) \\
\text { Mean gravel percentage }(\%) \\
\text { Density range (ind/ 10L) } \\
\text { Mean density (ind/ 10L) } \\
\text { Species richness } \\
\text { Diversity range } \\
\text { Mean diversity } \\
\text { Number of samples } \\
\text { Characteristic species }\end{array}$ & $\begin{array}{c}732-1568 \\
1068 \pm 226 \\
3.0-6.2 \\
4.38 \pm 0.99 \\
8.26-19.90 \\
13.77 \pm 2.76 \\
20.60-100.00 \\
92.99 \pm 19.28 \\
0.00-79.40 \\
5.74 \pm 18.38 \\
0.00-38.7 \\
1.27 \pm 6.73 \\
0.00-25.71 \\
3.74 \pm 8.13 \\
69 \\
0.00-4.19 \\
1.76 \pm 1.22 \\
33 \\
\text { Califia calida } \\
\text { Tharyx sp } \\
\text { Phimicolus } \\
\text { Neoheteromastus lineus } \\
\text { Maldane cristata } \\
\text { Maldanidae indeterminable } \\
\text { Scalibregma inflatum } \\
\text { Onuphis iridescens } \\
\text { Lumbrineridae indeterminable } \\
\text { Sternaspis fossor } \\
\text { Brada pluribranchiata } \\
\text { Fauveliopsis rugosa } \\
\text { Melinna exilia } \\
\text { Melinnampharete eoa } \\
\text { Mexamage corrugata } \\
\text { Thelepus hamatus }\end{array}$ & $\begin{array}{c}1230-2110 \\
1652 \pm 317 \\
2.1-3.7 \\
2.77 \pm 0.48 \\
10.27-15.47 \\
13.57 \pm 1.76 \\
92.70-100.00 \\
97.36 \pm 2.90 \\
0.00-7.30 \\
2.64 \pm 2.90 \\
0.00 \\
0.00 \pm 0.00 \\
0.00-7.60 \\
1.65 \pm 2.13 \\
43 \\
2.00-3.49 \\
2.73 \pm 0.57 \\
9 \\
\text { Califia calida } \\
\text { Phyllochaetopterus limicolus } \\
\text { Chaetozone setosa } \\
\text { Aphelochaeta monilaris } \\
\text { Tharyx sp } \\
\text { Neoheteromastus lineus } \\
\text { Maldanidae sp } 3 \\
\text { Maldanidae sp } 5 \\
\text { Maldanidae indeterminable } \\
\text { Exogone lourei } \\
\text { Goniada brunnea } \\
\text { Onuphis profundi } \\
\text { Lumbrineridae indeterminable } \\
\text { Ampharete arctica } \\
\text { Melinna exilia } \\
\text { Samythella pala } \\
\text { Terebellides stroemi }\end{array}$ & $\begin{array}{c}2032 \\
2032 \pm 0 \\
2.0 \\
2.00 \pm 0.00 \\
14.96 \\
14.96 \pm 0.00 \\
99.20 \\
99.20 \pm 0.00 \\
0.80 \\
0.80 \pm 0.00 \\
0.00 \\
0.00 \pm 0.00 \\
0.43 \\
0.43 \pm 0.00 \\
7 \\
2.87 \\
2.87 \pm 0.00 \\
1 \\
\text { Phylo nudus } \\
\text { Notomastus sp } \\
\text { Maldanidae sp } 2 \\
\text { Ancistrosyllis hartmanae } \\
\text { Nephtys paradoxa } \\
\text { Samythella pala } \\
\text { Ampharetidae sp }\end{array}$ \\
\hline
\end{tabular}

TABLE 6. - Ranges and mean values of the biotic and abiotic variables and characteristic species of each temperature group.

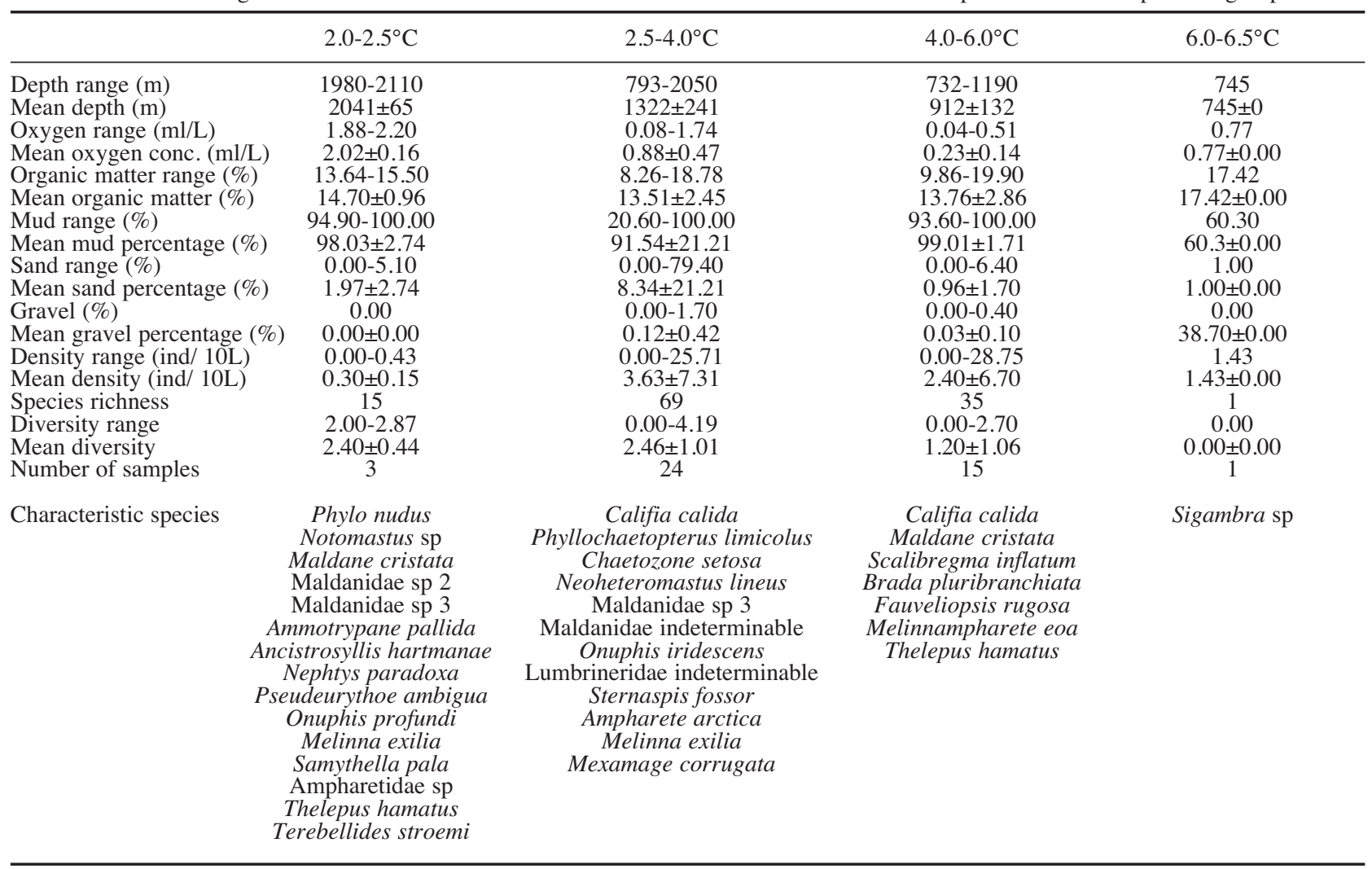


TABLE 7. - Ranges and mean values of the biotic and abiotic variables and characteristic species of each organic matter content group.

\begin{tabular}{|c|c|c|c|}
\hline & $8-16 \%$ & $16-18 \%$ & $18-20 \%$ \\
\hline $\begin{array}{l}\text { Depth range }(\mathrm{m}) \\
\text { Mean depth }(\mathrm{m}) \\
\text { Oxygen range }(\mathrm{ml} / \mathrm{L}) \\
\text { Mean oxygen conc. }(\mathrm{ml} / \mathrm{L}) \\
\text { Temperature range }\left({ }^{\circ} \mathrm{C}\right) \\
\text { Mean temperature }\left({ }^{\circ} \mathrm{C}\right) \\
\text { Mud range }(\%) \\
\text { Mean mud percentage }(\%) \\
\text { Sand range }(\%) \\
\text { Mean sand percentage }(\%) \\
\text { Gravel }(\%) \\
\text { Mean gravel percentage }(\%) \\
\text { Density range (ind/ 10L) } \\
\text { Mean density (ind/ 10L) } \\
\text { Species richness } \\
\text { Diversity range } \\
\text { Mean diversity } \\
\text { Number of samples }\end{array}$ & $\begin{array}{c}789-2110 \\
1261 \pm 348 \\
0.04-2.20 \\
0.79 \pm 0.60 \\
2.00-5.90 \\
3.79 \pm 1.03 \\
20.60-100.00 \\
94.29 \pm 16.91 \\
0.00-79.40 \\
5.63 \pm 16.90 \\
0.00-1.70 \\
0.08 \pm 0.33 \\
0.00-25.71 \\
4.09 \pm 8.20 \\
81 \\
0.00-4.19 \\
2.19 \pm 1.03 \\
39\end{array}$ & $\begin{array}{c}732-785 \\
765 \pm 28 \\
0.12-0.77 \\
0.35 \pm 0.37 \\
5.50-6.20 \\
5.77 \pm 0.38 \\
60.30-100.00 \\
86.77 \pm 22.92 \\
0.00-1.00 \\
0.33 \pm 0.58 \\
0.00-38.70 \\
12.90 \pm 22.34 \\
0.00-3.68 \\
1.81 \pm 1.72 \\
3 \\
0.00 \\
0.00 \pm 0.00 \\
3\end{array}$ & $\begin{array}{c}775-793 \\
784 \pm 12 \\
0.19-0.20 \\
0.20 \pm 0.01 \\
3.60-5.80 \\
4.70 \pm 1.56 \\
98.80-99.40 \\
99.10 \pm 0.42 \\
0.60-1.20 \\
0.89 \pm 0.41 \\
0.00 \\
0.00 \pm 0.00 \\
0.00-1.19 \\
1.19 \pm 0.00 \\
2 \\
0.00-1.00 \\
0.50 \pm 0.71 \\
2\end{array}$ \\
\hline Characteristic species & $\begin{array}{c}\text { Califia calida } \\
\text { Phyllochaetopterus limicolus } \\
\text { Tharyx sp } \\
\text { Neoheteromastus lineus } \\
\text { Maldane cristata } \\
\text { Maldanidae indeterminable } \\
\text { Onuphis iridescens } \\
\text { Lumbrineridae indeterminable } \\
\text { Melinna exilia } \\
\text { Melinnampharete eoa } \\
\text { Mexamage corrugata }\end{array}$ & $\begin{array}{l}\text { Sigambra sp } \\
\text { Ceratocephale pacifica } \\
\text { Brada pluribranchiata }\end{array}$ & $\begin{array}{l}\text { Chaetozone setosa } \\
\text { Ninoe fuscoides }\end{array}$ \\
\hline
\end{tabular}

TABLE 8. - Ranges and mean values of the biotic and abiotic variables and characteristic species of each mud percentage group.

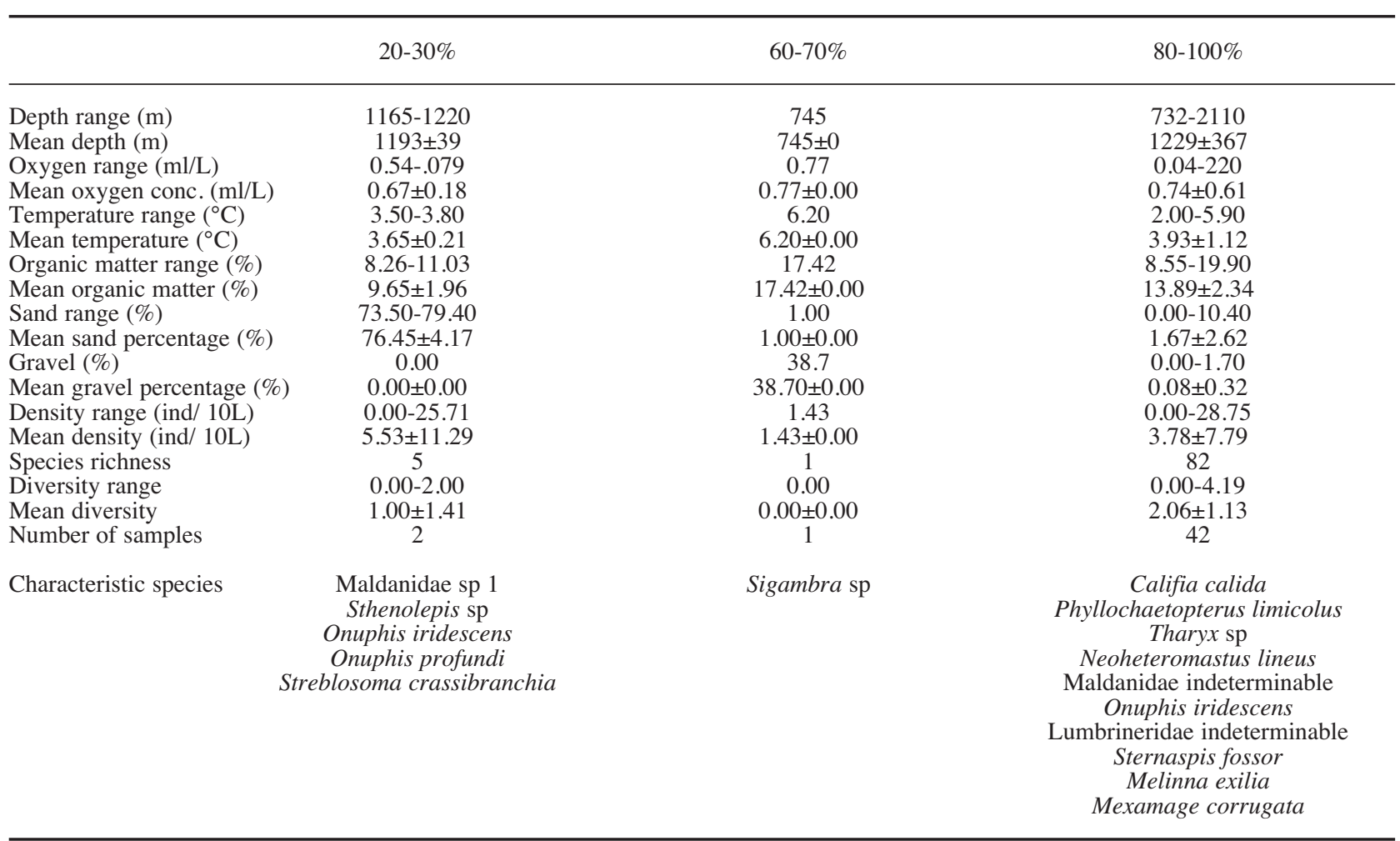




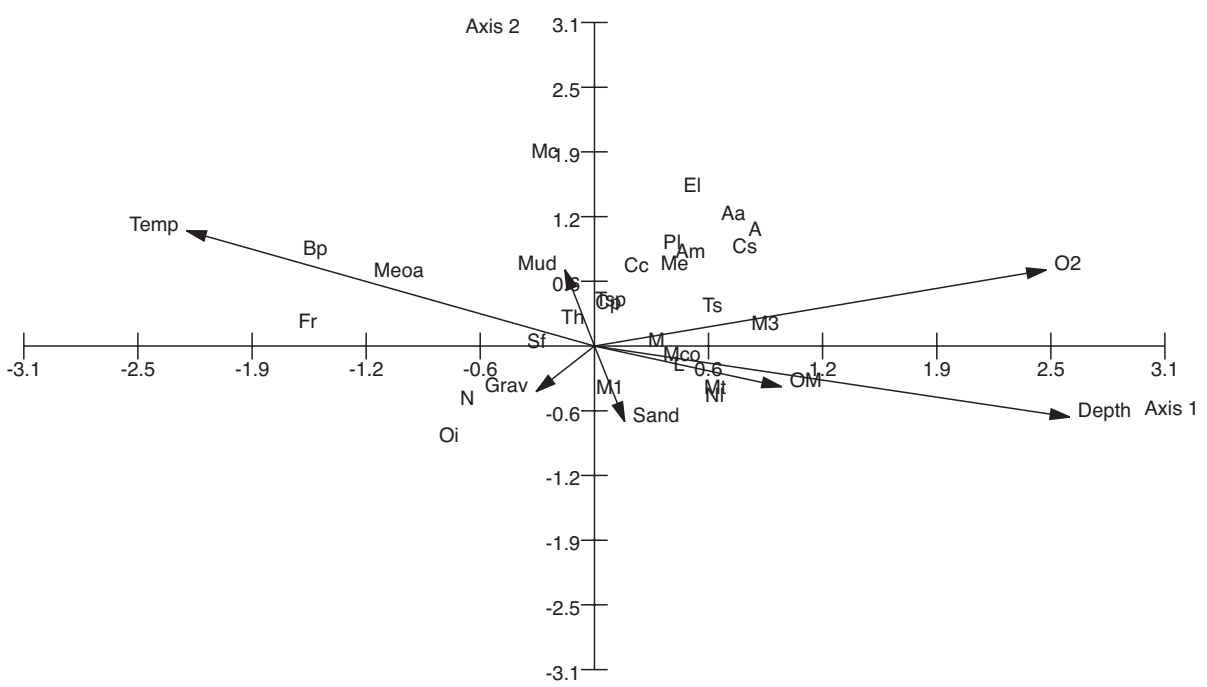

FIG. 7. - Canonical correspondence analysis ordination showing the 26 dominant and frequent species with respect to the abiotic variables, relative to axes 1 and 2 (Temp= temperature; $O 2=$ dissolved oxygen; $O M=$ organic matter; Grav= gravel; A= Ampharetidae indeterminable; $\mathrm{Aa}=$ Ampharete arctica $; \mathrm{Am}=$ Aphelochaeta multifilis; $\mathrm{Bp}=$ Brada pluribranchiata; $\mathrm{Cc}=$ Califia calida $; \mathrm{Cp}=$ Ceratocephale pacifica; $\mathrm{Cs}=$ Chaetozone setosa $; \mathrm{El}=$ Exogone lourei $; \mathrm{Fr}=$ Fauveliopsis rugosa $; \mathrm{L}=$ Lumbrineridae indeterminable; $\mathrm{M}=\mathrm{Maldanidae}$ indeterminable; $\mathrm{Mc}=$ Maldane cristata $; \mathrm{Mco}=$ Mexamage corrugata $; \mathrm{Me}=$ Melinna exilia $; \mathrm{Meoa=Melinnampharete} \mathrm{eoa} ; \mathrm{M} 1=$ Maldanidae sp 1; M3= Maldanidae sp 3; $\mathrm{N}=$ Nereidae indeterminable $; \mathrm{Nl}=$ Neoheteromastus lineus $; \mathrm{O}=$ Onuphis iridescens $; \mathrm{Pl}=$ Phyllochaetopterus limicolus $; \mathrm{Sf}=$ Sternaspis fossor $; \mathrm{Th}=$ Thelepus hamatus; $\mathrm{Ts}=$ Terebellides stroemi $; \mathrm{Tsp}=$ Thary $x \mathrm{sp} ; \mathrm{T} \mathrm{t}=$ Monticellina tesselata $)$.
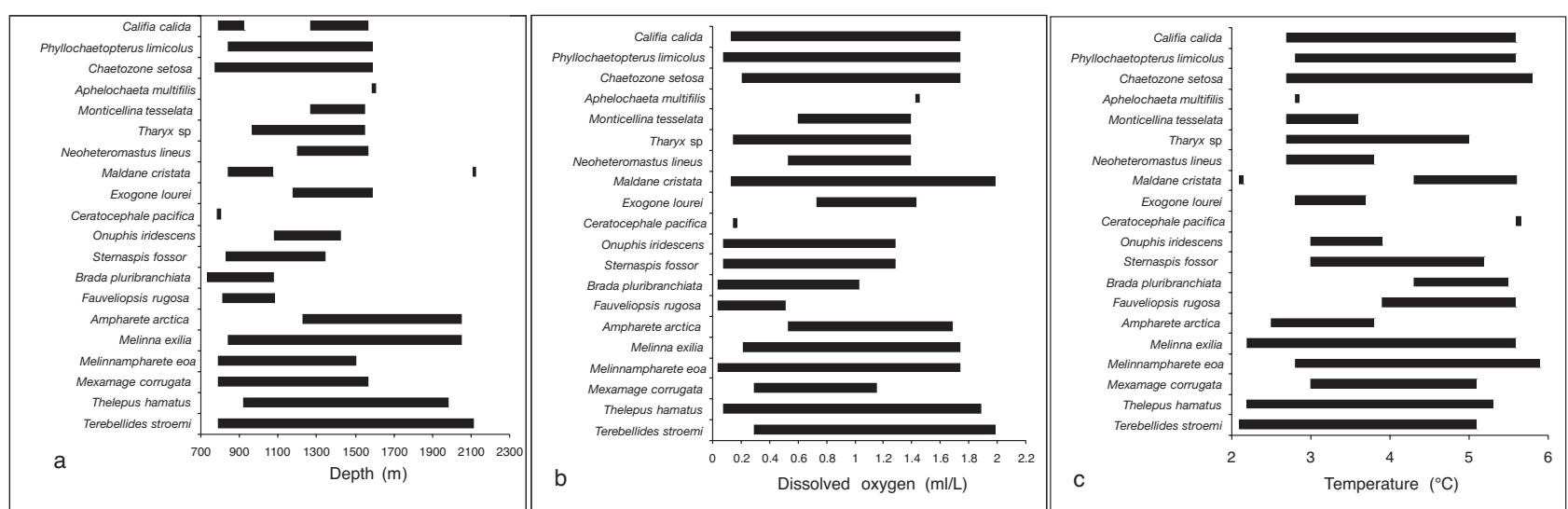

FIG. 8. - Depth (a), dissolved oxygen (b), and temperature (c) associated with the identified dominant and frequent species over the study.

\section{DISCUSSION}

All the species have been previously collected in the deep sea (abyssal or canyon depths), except for Halosydna johnsoni (Hartman, 1939, 1968; Berkeley and Berkeley, 1939, 1941; Rioja, 1962; Kudenov, 1975), and Ancistrosyllis hartmanae (Pettibone, 1966; Fiege and Bögemann,1999), which have only been found in shallow waters. Except for the pilargid Ancistrosyllis hartmanae and the ampharetid Melinnampharete eoa, all the species identified during this study have been previously reported for the Gulf of California or other areas in the eastern Pacific by Hartman $(1957,1960,1963$, 1968, 1969), Pettibone (1966), Fauchald (1968,
1970, 1972), Fauchald and Hancock (1981), or Petersen (2000).

Recent studies concerning deep-sea macrofauna (Levin and Gage, 1998; Levin et al., 2000, Levin et al., 2001, Levin, 2003, among others) have proposed the use of a $300-\mu \mathrm{m}$ mesh size instead of the traditional $500 \mu \mathrm{m}$. The use of a $500-\mu \mathrm{m}$ mesh could result in the loss of small specimens normally found in deep-sea samples, such as dorvilleids (Levin, pers. com.; May, 2005). Moreover, analyses of stomach contents from the fish Cherublemma emmelas (Gilbert, 1890) collected during the cruises of this study revealed the presence of small species of paranoids and pilargids, which were not found in our samples. 
It has been demonstrated that depth, oxygen and organic matter strongly influence the diversity of bathyal macrobenthos (Levin and Gage, 1998), depth being one of the most important factors affecting macrofauna distribution (Jumars, 1976; Grassle, 1989), as demonstrated by results obtained in the CCA.

\section{Depth}

The North Atlantic, the most intensively sampled region of the deep sea, has unimodal diversity-depth patterns, with a peak at intermediate depths and depressed diversity at upper bathyal and abyssal depths; however, this pattern does not appear to be universal (Levin et al., 2001). Results found here suggest a similar pattern since diversity increased with depth and reached a maximum value of 4.194 at $1270 \mathrm{~m}$, and then decreased slightly with depth. The minimum diversity value was 2.000 at $2110 \mathrm{~m}$ depth (Table 2). Due to the scarcity of samples in water deeper than $1270 \mathrm{~m}$ (only 13), a possible similitude between our study area and the North Atlantic zone could not be established.

The dominant families were represented in all the bathymetric assemblages, so a clear polychaete family representation in the different depth assemblages related to the OMZ position such as that described by Levin et al. (2000) in the NW Arabian Sea was not observed.

In this study, four faunal bathymetric assemblages were established based on polychaete density. The zone comprised of samples from 1100 to $1600 \mathrm{~m}$ had the highest number of species, density and diversity values, with a mean dissolved oxygen value of 0.87 $\mathrm{ml} / \mathrm{L}$ (Table 4). According to Hendrickx (2001), in the same area the highest number of species of decapod crustaceans were caught in the 1188-1245 m depth range, where hypoxic $(0.6$ to $0.76 \mathrm{ml} / \mathrm{L})$ conditions prevailed; the highest abundance of pandalid shrimps occurred between 1000 and $1500 \mathrm{~m}$ (Hendrickx, 2003a), and the highest abundance of the Pacific lobsterette Nephropsis occidentalis occurred between 1050 and $1310 \mathrm{~m}$. These depth ranges featured severe hypoxic conditions $(0.38-1.4 \mathrm{ml} / \mathrm{L})$, as stated by Hendrickx (2003b).

Although the 700-1100 $\mathrm{m}$ assemblage had the lowest oxygen values (a mean of $0.28 \mathrm{ml} / \mathrm{L}$ ), it showed a relatively high diversity, species number and density. On the other hand, the 1900-2100 m and 2100-2200 $\mathrm{m}$ assemblages contained the highest dissolved oxygen levels (means of 1.92 and 1.98 $\mathrm{ml} / \mathrm{L}$, respectively), but had low values for the biotic variables. A similar situation was reported in the same area by Hendrickx (2001), who discovered that sampling stations with higher oxygen content (above $2.0 \mathrm{ml} / \mathrm{L}$ at depths greater than $2000 \mathrm{~m}$ ) were inhabited by fewer benthic decapod species. He concluded that, although critically low oxygen content $(<0.5 \mathrm{ml} / \mathrm{L})$ may represent an impediment for the establishment of a rich benthic fauna, a higher oxygen content does not necessarily favour the presence of a richer fauna.

\section{Dissolved oxygen}

According to Levin (2003), density of macrofauna is often depressed within the part of the $\mathrm{OMZ}$ where the lowest oxygen concentrations occur. Results found here, however, did not show a significant correlation between oxygen and density. Thus, other biotic variables such as species richness or diversity could explain the relationship between polychaete composition and oxygen concentration. The OMZs have been defined as regions where bottom-water oxygen concentrations are $<0.5 \mathrm{ml} / \mathrm{L}$ and are characterised by low species richness and very high dominance. This pattern has been reported on the West African margin (Sanders, 1969), in the eastern Pacific, on a seamount off Mexico (Levin et $a l ., 1991)$, in the NW Arabian Sea off Oman (Levin et al., 1997; Levin et al., 2000), and on the Peru and Chile margins (Levin et al., 2002). Analyses performed by Levin and Gage (1998) with samples from several localities suggested a positive relationship between bottom-water oxygen concentration and species richness. The same situation was observed in this study, since oxygen had a significant positive correlation with species number (Table $3)$. The highest number of species was found in the $0.00-2.00 \mathrm{ml} / \mathrm{L}$ assemblage (Table 5), but this group included $76.7 \%$ of the samples. Levin et al. (2001) suggested that a significant reduction of macrofaunal species richness in a low oxygen environment may not occur until the concentration falls below 0.4 or $0.3 \mathrm{ml} / \mathrm{L}$. Such a reduction could be even lower for annelids, due to their high tolerance to hypoxia. Data registered here indicate a different situation, since species richness in samples below 0.3-0.4 $\mathrm{ml} / \mathrm{L}$ ranged from 1 to 10 species, similar to samples with higher oxygen concentrations (Table 2).

Studies by Sanders (1969) off West Africa, by Levin et al. (1991) in the eastern Pacific, and by 
Levin et al. (1997) in the Arabian Sea have shown extreme depression of macrobenthic diversity within OMZs, beneath upwelling regions, where organic matter flux to the seabed is high. Here, a significant positive correlation was found between diversity and oxygen concentration and, in general terms, the low-oxygen group showed the lowest mean diversity (Table 5). Nevertheless, a closer analysis indicates that stations where diversity was null had oxygen concentrations ranging from 0.10 to $0.79 \mathrm{ml} / \mathrm{L}$ without a clear pattern (Table 2). Thus, for the southeastern Gulf of California, it can be concluded that it is difficult to distinguish the importance of oxygen stress in determining species richness and diversity changes associated to OMZs.

Studies on macrobenthos in the Indo-Pacific and Atlantic oceans have suggested that there is an oxygen threshold (i.e. $1 \mathrm{ml} / \mathrm{L}$ or maybe $0.45 \mathrm{ml} / \mathrm{L}$ ) above which oxygen has little influence on species richness, but below which it is a powerful control (Levin and Gage, 1998). Moreover, in the Oman margin, Levin et al. (2000) concluded that maxima in abundance, biomass and annelid body size encountered at $700 \mathrm{~m}(0.16 \mathrm{ml} / \mathrm{L})$ may reflect a threshold release from oxygen stress in enriched sediments. We did not recognize clear OMZ thresholds based on deep-water polychaete composition, since grouping of stations each $0.4 \mathrm{ml} / \mathrm{L}$ included wide oxygen ranges and no clear gradients were observed. Furthermore, some species were characteristic of several oxygen groups. A similar situation occurred with the mollusc community in the same study area (Zamorano et al., 2006), where no clear oxygen groups could be established. In addition, polychaete density, diversity and species richness did not show clear patterns associated with specific oxygen concentrations. To observe a possible low oxygen threshold, samples with oxygen $<0.21 \mathrm{ml} / \mathrm{L}$ were closely analysed. The average species richness was $2.58 \pm 2.19$ (SD), but the 12 samples contained 20 species, each one present in at most 3 samples. Moreover, diversity ranged from 0 to 2.922 (average: $0.871 \pm 0.941, \mathrm{SD})$ and density, from 0 to 26.973 (average: $7.43 \pm 11.47, \mathrm{SD}$ ). This confirmed that oxygen is not the main abiotic variable affecting polychaete distribution in the area.

\section{Temperature}

The correlation values and results from the CCA obtained here suggest that temperature is also an important factor controlling polychaete composition. Narayanaswamy et al. (2003) and Narayanaswamy et al. (2005) stated the importance of temperature in deep-sea polychaete distributions. The last work was performed in stations located between 350 and $550 \mathrm{~m}$ in the north-east Atlantic. Hernández-Alcántara and Solís-Weiss (2005) found a strong effect of temperature on spionids from the Gulf of California in the sublittoral zone, between 22 and $120 \mathrm{~m}$ depth. Results found here confirm these findings and extend them to deeper water zones, since significant negative correlations were found between temperature and the three biotic variables studied (Table 3). The faunal assemblages defined according to temperature suggest that 2.5 to $4.0^{\circ} \mathrm{C}$ was the range preferred by polychaetes, since it included higher density, diversity and species numbers than the other ranges (Table 6). However, this range does not seem to be exclusive for some of the characteristic species.

A closer approach considering only the dominant and frequent species over the study area indicates that these species were collected in wide temperature ranges, but some of them were found in restricted temperature, oxygen and depth ranges (Fig. 8). This suggests that the distribution of polychaetes in this area is directly affected by the combination of the three abiotic variables, which are strongly correlated with each other, as suggested by Wishner $e t$ al. (1995) for pelagic and benthic communities on a seamount off Acapulco. This explains the position of these species in the CCA in relation to the abiotic variables. For instance, considering the temperature $\left(2-6.2^{\circ} \mathrm{C}\right)$, oxygen $(0.04-2.2 \mathrm{ml} / \mathrm{L})$ and depth $(732-$ $2110 \mathrm{~m}$ ) ranges found throughout this study, $B$. pluribranchiata and $F$. rugosa live in high temperatures and low depths and oxygen contents and, conversely, A. arctica was found in deeper zones with higher oxygen content and lower temperatures (Figs. 7 and 8).

\section{Organic matter content}

Organic matter has been considered an important factor affecting deep-water polychaete distribution. Cook et al. (2000), Gooday et al. (2000), and Gooday et al. (2001) concluded that annelids often demonstrate parallel trends along gradients of oxygen and organic matter contents. In this study, the correlation between the two abiotic variables was non-significant (Table 3), probably due to the fact 
that organic matter content was always high and homogeneous over the study area; $88.6 \%$ of the samples were comprised in the $8-16 \%$ organic matter group (Table 7). The MDS plots did not suggest that polychaete species are distributed over organic matter or oxygen gradients (Figs. $3 b$ and $5 b$ ). Combined with the results obtained in the CCA, this suggests that organic matter content is not a limiting factor for deep-water polychaetes in the area, contrary to what was observed in the North Atlantic, the eastern Pacific, and the Indian Ocean, where organic carbon was found to strongly influence polychaete diversity (Levin and Gage, 1998).

The importance of organic matter content in sediments for macrofaunal diversity and species richness has been widely established. Levin et al. (1994) found reduced diversity at the most enriched sites from bathyal bottoms in North Carolina and on a Pacific seamount. In shallow waters, Pearson and Rosenberg (1978) found increasing polychaete diversity with increasing organic nutrients up to an intermediate level, and then a decline in diversity with even greater organic inputs. According to Levin and Gage (1998), increasing levels of organic matter may lead to reduced species richness or high dominance. The percentages of organic matter content from the southeastern Gulf of California are higher than data reported elsewhere, and this could be partially due to the technique used, which could overestimate the results. Nevertheless, these results are useful to observe tendencies. Similar diversity and species richness decreases were observed in the groups with higher organic matter content (Table 7), but without a clear pattern; null diversity values and the lowest species numbers ( 0 and 1$)$ were found in samples with percentages of organic matter between 8.26 and $18.78 \%$ (Table 2). In addition, none of the stations was dominated by one or two species, which also reduces the importance of this variable throughout the study area. It is therefore difficult to generalize about organic-matter influence on deepsea diversity, since this parameter varies in the different regions of the world, as stated by Levin and Gage (1998).

\section{Sediment characteristics}

Organic matter in sediments is strongly influenced by particle size (Milliman, 1994), as observed by the high correlation between percentage of mud and organic matter (Table 3). The granulometry and organic matter content analyses indicated that the sediment characteristics were relatively homogeneous throughout the study area, since mud dominated at most of the stations (i.e. a single group included $95.5 \%$ of the stations; Table 8 ) and organic matter was always high (from 8.26 to $18.78 \%$ ). No clear gradients of faunal distribution were observed according to these variables (Figs. 5b and 6b). Consequently, these variables were less important than depth, temperature and oxygen in the CCA. The lack of importance of grain size in this study is supported by similar results obtained in the North Atlantic, the eastern Pacific and the Indian Ocean, where percentages of sand and clay were considered as non-significant factors affecting macrofaunal distribution (Levin and Gage, 1998).

Endo-benthic fauna distribution depends strongly on sediment characteristics, due to the building of structures such as tubes and burrows (grain size) and as a food supply (organic matter) for detritivores (Wieser, 1959; Rhoads, 1974; Gray, 1981). According to Jumars (1976), large detritivores are favoured in the deep sea where organic matter content is high. Most of the dominant and frequent species of this study belong to the surface-deposit feeder category as proposed by Fauchald and Jumars (1979) and Gambi and Giangrande (1985). The high amounts of organic matter indicate that the deep-sea polychaete fauna in the southeastern Gulf of California is not food-limited, which also explains the lack of importance of this variable in the CCA.

The family Ampharetidae (surface feeders) was the best represented in terms of number of species, dominance and frequency. It has been previously reported that density of ampharetids increases with depth and the family is dominant in abyssal sediments (Day, 1967; Fauchald and Jumars, 1979; Hernández-Alcántara and Solís-Weiss, 1993). Fauchald and Jumars (1979) have demonstrated that some deep-water polychaetes build strong ventilating tubes to tolerate low oxygen conditions, and the continuous tube building of ampharetids may represent a form of locomotion. Most of the collected ampharetid tubes were large (up to $30 \mathrm{~cm}$ long), wide (up to $1 \mathrm{~cm}$ ) and robust (Méndez, 2006), which explains the success of this family in the study area.

Maldanids and cirratulids were also dominant and frequent. According to Fauchald and Jumars (1979) and Gambi and Giangrande (1985), most maldanids are tubicolous and are generally represented by subsurface-deposit feeders. The high 
organic matter content in sediment as well as the dominance of compact mud explains the success of members of this family in this environment. Cirratulids are also classified as deposit feeders and are generally considered as non-selective worms. Cirratulids (Levin et al., 2000) and many species from the genus Tharyx (Jumars, 1975) are among the most abundant macrofaunal species in deep-sea, and this was confirmed in this study.

Surface and subsurface-deposit feeding is likely to be important for species living in bathyal and abyssal regions (Fauchald and Jumars, 1979). This is the case of the dominant and frequent species belonging to the tubicolous families Chaetopteridae, Capitellidae and Terebellidae and the non-tubicolous Orbiniidae, Flabelligeridae, Syllidae and Sternaspidae. Few species belonging to predominantly carnivorous (Lumbrineridae) and omnivorous (Nereidae and Onuphidae) families were dominant or frequently present. These species do not compete for food with detritivores, allowing for equilibrium in the deep sea.

Results from this study showed that deep-water polychaetes from the southeastern Gulf of California do not distribute following clear temperature, depth, oxygen, organic matter or sediments grain size gradients. Species were not found in specific ranges of these variables, thus making it difficult to establish clear distribution patterns based on polychaete analyses and suggesting that none of these variables is the key factor controlling polychaete distribution in this zone. Several deep-sea studies performed in different regions of the world (i.e. Rosenberg et al., 1991; Etter and Grassle, 1992; Diaz and Rosenberg, 1995; Wishner et al., 1995; Levin and Gage, 1998; Levin et al., 2000; Rex et al., 2000; Levin, 2001, Hendrickx, 2003a; Narayanaswamy, et al., 2003; Narayanaswamy, et al., 2005; Levin et al., 2001) have revealed that bathyal macrofaunal density, species richness and diversity are strongly influenced by dissolved oxygen concentration and depth. However, these studies also indicate that other factors such as temperature, organic matter in sediments, grain size, latitude, and sediment pigments are important parameters influencing macrofaunal distribution. It is now demonstrated that deep-sea polychaete distribution in the southeastern Gulf of California is controlled by the combination of several environmental variables, the most important being depth, oxygen concentration and temperature, whose effects can be variable in the different world's oceans, as mentioned by Levin (2003).

\section{CONCLUSIONS}

This study is a contribution to the scarce knowledge of the influence of environmental parameters on the distribution of deep-sea polychaetes inhabiting the southeastern Gulf of California. The density of polychaetes collected during the four oceanographic cruises in a depth range from 732 to $2110 \mathrm{~m}$ allowed faunal assemblages to be established according to the studied environmental variables. Polychaete distribution was strongly associated with depth, dissolved oxygen and temperature. Grain size and organic matter contents in sediment had a minor contribution since those variables remained homogeneous throughout the study area. World-wide deep-sea studies previously described indicate that polychaete distribution patterns are associated with a combination of several environmental factors, whose effects vary in the different geographic zones. Thus, further detailed investigations are strongly recommended in specific regions of the world's oceans.

\section{ACKNOWLEDGEMENTS}

This study was supported by the CONACyT 31805-N and PAPIIT IN217306-3 projects. I wish to thank Michel Hendrickx, director of these projects, for his advice and for reviewing the manuscript. I am especially grateful to Lisa Levin for her useful suggestions and comments on data interpretation, and her thorough revision of the manuscript. Samples were collected by the crew members of the R/V "El Puma", and students and technical staff from the Unidad Académica Mazatlán (Instituto de Ciencias del Mar y Limnología, UNAM). I am also grateful to Arturo Toledano, José Salgado, Héctor Nava, Benjamín Yáñez, Ernesto Sánchez and Alejandro Nava for their help during sampling. José Luis Carballo helped with statistics and Germán Ramírez and David Serrano in drawing up the figures.

\section{REFERENCES}

Alvarez-Borrego, S. and R.A. Schwartzlose. - 1979. Masas de agua del Golfo de California. Cienc. Mar., 6: 43-63.

Berkeley, E. and C. Berkeley. - 1939. On a collection of Polychaeta, chiefly from the West Coast of Mexico. Ann. Mag. N. Hist. Ser. 11, 3: 321-346.

Berkeley, E. and C. Berkeley. - 1941. On a collection of Polychaeta from Southern California. Bull. So. Calif. Acad. Sci., 40: 16-59.

Blake, J.A. - 1985. Polychaeta from the vicinity of deep-sea geot- 
hermal vents in the eastern Pacific I: Euphrosinidae, Phyllodocidae, Hesionidae, Nereidae, Glyceridae, Dorvilleidae, Orbiniidae, and Maldanidae. Bull. Biol. Soc. Wash., 6: 67-101.

Blake, J.A. and B. Hilbig. - 1990. Polychaeta from the vicinity of deep-sea hydrothermal vents in the eastern Pacific. II. New species and records from the Juan de Fuca and Explorer ridge systems. Pac. Sci., 44: 219-253.

Blake, J.A., J.A.. Muramoto, B. Hilbig and I.P. Williams. - 1992. Biological and sedimentological investigations of the sea floor at the proposed U.S. Navy Ocean disposal site. July 1991 survey $(R / V$ Wecoma $)$. Benthic biology and sediment characterization. Final Report submitted to PRC Environmental Management, Inc., Honolulu, Hawaii. iii+130 pp., Appendices A-D.

Blake, J.A. and B. Hilbig. - 1994. Taxonomic Atlas of the Santa Maria Basin and Western Santa Barbara Channel. Vol. 4. Annelida Part 1. Oligochaeta and Polychaeta (Phyllodocidae to Paralacydoniidae). Santa Barbara Museum of Natural History.

Blake, J.A., B. Hilbig and P.H. Scott. - 1995. Taxonomic Atlas of the Santa Maria Basin and Western Santa Barbara Channel. Vol. 5. Annelida Part 2. Polychaeta (Phyllodocida, Amphinomida and Eunicida). Santa Barbara Museum of Natural History.

Blake, J.A., B. Hilbig and P.H. Scott. - 1996. Taxonomic Atlas of the Santa Maria Basin and Western Santa Barbara Channel. Vol. 6. Annelida Part 3. Polychaeta (Orbiniidae to Cossuridae). Santa Barbara Museum of Natural History.

Blake, J.A., B. Hilbig and P.H. Scott. - 2000. Taxonomic Atlas of the Santa Maria Basin and Western Santa Barbara Channel. Vol. 7. Annelida Part 4. Polychaeta (Flabelligeridae to Ampharetidae). Santa Barbara Museum of Natural History.

Chamberlin, R.V. - 1919. New polychaetous annelids from Laguna Beach, California. J. Ent. \& Zool., 11: 1-23.

Cook, A.A., P.J.D. Lambshead, L.E. Hawkins, N. Mitchell and L.A Levin. - 2000. Nematode abundance at the oxygen minimum zone in the Arabian Sea. Deep-Sea Res. Pt. II, 47: 75-85.

Day, J.H. - 1967. A monograph on the polychaeta of Southern Africa. Part I: Errantia. Part II: Sedentaria. British. Museum (Natural History), 656: 1-878.

Dean, W.E., Jr. - 1974. Determination of carbonate and organic matter in calcareous sediments and sedimentary rocks by loss of ignition: comparison with other methods. J. Sed. Petrol., 44: 242-248.

Diaz, R.J. and R. Rosenberg. - 1995. Marine benthic hypoxia: a review of its ecological effects and the behavioural responses of benthic macrofauna. Oceanogr. Mar. Biol. Annual Rev., 33 245-303.

Etter, R.J. and J.F. Grassle. - 1992. Patterns of species diversity in the deep sea as a function of sediment particle size diversity. Nature, 360: 576-578.

Fauchald, K. - 1968. Onuphidae (Polychaeta) from Western Mexico. Allan Hancock Monogr. Mar. Biol., 3: 1-82.

Fauchald, K. - 1970. Polychaetous annelids of the families Eunicidae, Lumbrineridae, Iphitimidae, Arabellidae, Lysaretidae and Dorvilleidae from western Mexico. Allan Hancock Monogr. Mar. Biol., 5: 1-335.

Fauchald, K. - 1972. Benthic polychaetous annelids from deep water off western Mexico and adjacent areas in the Eastern Pacific Ocean. Allan Hancock Monogr. Mar. Biol., 7: 1-575.

Fauchald, K. - 1977. The polychaete worms. Definitions and keys to the orders, families and genera. Natural History Museum of Los Angeles County, and Allan Hancock Foundation. Science Series 8: 1-188.

Fauchald, K. and P.A. Jumars. - 1979. The diet of worms: a study of polychaete feeding guilds. Oceanogr. Mar. Biol. Annual Rev., 17: 193-284

Fauchald, K. and D.R. Hancock. - 1981. Deep-water polychaetes from a transect off central Oregon. Allan Hancock Found. Monogr. Mar. Biol., 11: 1-73.

Fiege, D. and M. Bögemann. - 1999. Ancistrosyllis fioronii, a new species of Pilargidae from the North Sea, with a key and synoptic table of characters for all species of the genus Ancistrosyllis McIntosh 1879 (Annelida, Polychaeta, Pilargidae). Senckenb. Biol., 78:135-140.

Field, J.G., K.R. Clarke and R.M. Warwick. - 1982. A practical strategy for analysing multispecies distribution patterns. Mar. Ecol. Progr. Ser., 8: 37-52.
Folk, R.L. - 1965. Petrology of Sedimentary Rocks. Hemphills Publications Company, Austin.

Gambi, M.C. and A. Giangrande. - 1985. Caratterizzazione e distribuzione delle catagorie trofiche dei policheti nei fondi mobili del golfo di Salerno. Oebalia, 11: 223-240.

Glémarec, M. - 1964. Bionomie benthique de la partie orientale du golfe du Morbihan. Cah. Biol. Mar., 5: 33-96.

Grassle, J.F. - 1989. Species diversity in deep-sea communities. Trends Ecol. Evol. 4: 12-15.

Gray, J.S. - 1981. The Ecology of Marine Sediments. An introduction to the Structure and Function of Benthic Communities. Cambridge University Press. Cambridge.

Gooday, A.J., J.M. Bernhard, L.A. Levin and S. Suhr. - 2000. Foraminifera in the Arabian Sea OMZ and other oxygen deficient settings: taxonomic composition, diversity and relation to metazoan faunas. Deep-Sea Res. Pt. II, 47: 54-73.

Gooday, A.J., J.A. Hughes and L.A. Levin. - 2001. The foraminiferal macrofauna from three North Carolina (U.S.A.) slope sites with contrasting carbon flux: a comparison with the metazoan macrofauna. Deep-Sea Res. Pt. I, 48: 1709-1739.

Hartman, O. - 1939. Polychaetous annelids. Part I. Aphroditidae to Pisionidae. Allan Hancock Pac. Exped., 7: 1-156.

Hartman, O. - 1944. Polychaetous annelids. Part V. Eunicea. Allan Hancock Pac. Exped., 10: 1-237.

Hartman, O. - 1957. Orbiniidae, Apistobranchidae, Paraonidae and Longosomidae. Allan Hancock Pac. Exped., 15: 207-393.

Hartman, O. - 1960. Systematic account of some marine invertebrate animals from the deep Basins off southern California. Allan Hancock Pac. Exped., 22: 69-215.

Hartman, O. - 1963. Submarine canyons of southern California Polychaetous annelids. Allan Hancock Pac. Exped., 27: 1-93.

Hartman, O. - 1968. Atlas of Errantiate Polychaetous Annelids from California. Allan Hancock Foundation. University of Southern California, Los Angeles. 828 pp.

Hartman, O. - 1969. Atlas of Sedentariate Polychaetous Annelids from California. Allan Hancock Foundation. University of Southern California, Los Angeles. 812 pp.

Hendrickx, M.E. - 2001. Occurrence of a continental slope decapod crustacean community along the edge of the minimum oxygen zone in the south eastern Gulf of California, México. Belg. J. Zool., 131: 95- 110.

Hendrickx, M.E. - 2003a. Size and abundance of deep water shrimps on the continental slope of the SE Gulf of California, México. In: M.E. Hendrickx (ed.), Contributions to the Study of East Pacific Crustaceans, pp. 227- 234. Instituto de Ciencias del Mar y Limnología, UNAM. Vol. 2.

Hendrickx, M.E. - 2003b. Distribution and size of the Pacific deep water lobsterette Nephropsis occidentalis Faxon, 1893 (Decapoda, Astacidea, Nephropidae) in the Gulf of California, México. Crustaceana, 76: 207-216.

Hernández-Alcántara, P. - 1992. Los poliquetos (Annelida: Polychaeta) de la plataforma continental del Golfo de California, México. Taxonomía Abundancia numérica y distribución geográfica. Masters Thesis, ICMyL, UNAM. México D.F.

Hernández-Alcántara, P. and V. Solís-Weiss. - 1993. Distribución latitudinal y batimétrica de los anélidos poliquetos del Orden Terebellomorpha de la plataforma continental del Golfo de California, México. Cuad. Mex. Zool., 1: 65-72.

Hernández-Alcántara. P. and V. Solís-Weiss. - 2005. Seasonal variations of the Spionida (Palpata: Canalipalpata) in the sublittoral zone of the Gulf of California. Mar. Ecol., 26: 273-285.

Jumars, P.A. - 1975. Target species for deep-sea studies in ecology, genetics, and physiology. Zool. J. Linn. Soc., 57: 341-348.

Jumars, P.A. - 1976. Deep-sea species diversity: does it have a characteristic scale? J. Mar. Res., 34: 217-246.

Kudenov, J.D. - 1975. Errant polychaetes from the Gulf of California, Mexico. J. Nat. Hist. London, 9: 65-91.

Levin, L.A., C.L. Huggett and K.F. Wishner. - 1991. Control of deep-sea benthic community structure by oxygen and organicmatter gradients in the eastern Pacific Ocean. J. Mar. Res., 49: $763-800$.

Levin, L.A., G.R. Plaia and C.L. Huggett. - 1994. The influence of natural organic enhancement on life histories and community structure of bathyal polychaetes. In: C.M. Young and K.J. Eckelbarger (eds.), Reproduction, Larval Biology, and Recruitment of the Deep-Sea Benthos. pp. 261-283. Columbia University Press, New York. 
Levin, L.A., J. Gage, P. Lamont, L. Cammidge, C. Martin, A. Patience and J. Crooks. - 1997. Infaunal community structure in a low-oxygen, organic rich habitat on the Oman Continental slope, NW Arabian Sea. In: L.E. Hawkins and S. Hutchinson (eds.), Responses of Marine Organisms to their Environments. Proceedings of the $30^{\text {th }}$ European Marine Biology Symposium. pp. 223-230. University of Southampton, Southampton.

Levin, L.A. and J.D. Gage. - 1998. Relationship between oxygen, organic matter and diversity of bathyal macrofauna. Deep-Sea Res. Pt. II., 45: 129-163.

Levin, L.A., J.D. Gage, C. Martin and P.A. Lamont. - 2000. Macrobenthic community structure within and beneath the oxygen minimum zone, NW Arabian Sea. Deep-Sea Res. Pt. II., 47: 189-226.

Levin, L.A. - 2001. Oxygen minimum zone influence on the community structure of deep-sea benthos. ASLO Meeting. Aquatic Sciences Meeting. Albuquerque, NM. February, 12-16 $6^{\text {th }}, 2001$. Abstracts.

Levin, L.A., J.E. Etter, M.A. Rex, A.J. Gooday, C.R. Smith, J. Pineda, C.T. Stuart, R.R. Hessler and D. Pawson. - 2001. Environmental influences on regional deep-sea species diversity. Ann. Rev. Ecol. Syst., 32: 51-93.

Levin, L.A., A.E. Rathburn, C. Neira, J. Sellanes, P. Muñoz, V. Gallardo and M. Salamanca. - 2002. Benthic processes on the Peru margin: A transect across the oxygen minimum zone during the 1997-1998 El Niño. Prog. Oceanogr., 53: 1-27.

Levin, L.A. - 2003. Oxygen minimum zone benthos: adaptation and community response to hypoxia. Oceanogr. Mar. Biol. Annual Rev., 41: 1-45.

Méndez, N. - 2006. Deep-water polychaetes (Annelida) from the southeastern Gulf of California, Mexico. Rev. Biol. Trop., 54: 773-785.

Milliman, J.D. - 1994. Organic matter content in U.S. Atlantic continental slope sediments: decoupling the grain-size factor. Deep-Sea Res. Pt. II., 41: 797-808.

Narayanaswamy, B.E., T.D. Nickell and J.D. Gage. - 2003. Appropriate levels of taxonomic discrimination in deep-sea studies: species vs family. Mar. Ecol. Progr. Ser., 257: 59-68.

Narayanaswamy, B.E., B.J. Bett and J.D. Gage. - 2005. Ecology of bathyal polychaete fauna at an Arctic-Atlantic boundary (Faroe-Shetland Channel, North-east Atlantic).Mar. Biol.Res., 1: 20-32.

Parker, R.H. - 1964. Zoogeography and ecology of some macroinvertebrates particularly mollusks, in the Gulf of California and the continental slope off Mexico. Vidensk. Medd. Fra Dansk Naturh., 126: 1-178.

Pearson, T.H. and R. Rosenberg. - 1978. Macrobenthic succesion in relation to organic enrichment and pollution of the marine environment. Oceanogr. Mar. Biol. Annual Rev., 16: 229-311.

Petersen, M.E. - 2000. A new genus of Fauveliopsidae (Annelida: Polychaeta), with a review of its species and description of some described taxa. Bull. Mar. Sci., 67: 491-515.

Pettibone, M.H. - 1966. Revision of the Pilargidae (Annelida: Polychaeta) including descriptions of new species, and redescription of the pelagic Podarmus ploa Chamberlin
(Polynoidae). Proc US Nat Mus. 118: 155-208

Picard, J. - 1965. Recherches qualitatives sur les biocénoses marines des substrats meubles dragables de la region marsellaise. Rec. trav. stn. mar. Endoume, 36: 1-60.

Rex, M.A., C.T. Stuart and G. Coyne. - 2000. Latitudinal gradients of species richness in the deep-sea benthos of the North Atlantic. P. Natl. Acad. Sci. USA, 97: 4082-4085.

Rhoads, C.D. -1974 . Organism-sediment relations on the muddy sea floor. Oceanogr. Mar. Biol. Annu. Rev., 12: 263-300.

Rioja, E. - 1962. Estudios anelidológicos. XXVI. Algunos anélidos poliquetos de las Costas del Pacífico de México. An. Inst. Biol. Mex., 33: 131-229.

Rosenberg, R., B. Hellman and B. Johansson. 1991. Hypoxic tolerance on marine benthic fauna. Mar. Ecol. Progr. Ser., 79: 127-131.

SAIC. - 1991. Biological and sedimentological investigations of the sea floor at the proposed U.S. Navy 103 Ocean Disposal Sit. Report submitted to PRC Environmental Management, Inc., San Francisco. 95 pp, Appendices A-C.

SAIC. - 1992. Benthic ecology and sediment characterization. Ocean studies report for detailed physical and biological oceanographic studies for an ocean site designation effort under the Marine Protection, Research and Sanctuaries Act of 1972 (MPRSA). Report prepared for U.S. Environmental Protection Agency, Region IX, San Francisco, CA.

Sanders, H.L. - 1969. Benthic marine diversity and the stabilitytime hypothesis. Brookhaven Symposia on Biology, 22: 71-81.

Schlieper, C. - 1972. Research methods in marine biology. Sidgwick and Jackson Biology Series, London.

Solís-Weiss, V. and B. Hilbig. - 1992. Redescription of Ophryotrocha platykephale Blake (Polychaeta, Dorvilleidae) from the Guaymas Basin hydrothermal vents. Bull. So. Calif. Acad. Sci., 91: 92-96.

Solís-Weiss, V. and P. Hernández-Alcántara. - 1994. Amphisamytha fauchaldi: a new species of Ampharetid (Annelida: Polychaeta) from the hydrothermal vents at Guaymas Basin, Mexico. Bull. So. Calif. Acad. Sci., 93: 127-134.

Strickland, J.D.H. and T.R. Parsons. - 1972. A Practical Handbook of Seawater Analysis. Fisheries Research Board of Canada, Bulletin 167 (second edition).

Wieser, W. - 1959. The effect of grain size on the distribution of small invertebrates inhabiting the beaches of Puget Sound. Limnol. Oceanogr., 4: 181-194.

Wishner, K.F., C.J. Ashjian, C. Gelfman, M.M. Gowing, L. Kann, L.A. Levin, L.S. Mulineaux and J. Saltzman. - 1995. Pelagic and benthic ecology of the lower interface of the Eastern Tropical Pacific oxygen minimum zone. Deep-Sea Res. Pt. I, 42: $93-115$

Zamorano, P., M.E. Hendrickx and A. Toledano-Granados. - 2006. Distribution and ecology of deep water mollusks from the continental slope, southeastern Gulf of California, Mexico. Mar. Biol., 150: 883-892.

Scient. ed.: J.E. Cartes.

Received January 26, 2007. Accepted May 25, 2007.

Published online August 3, 2007. 